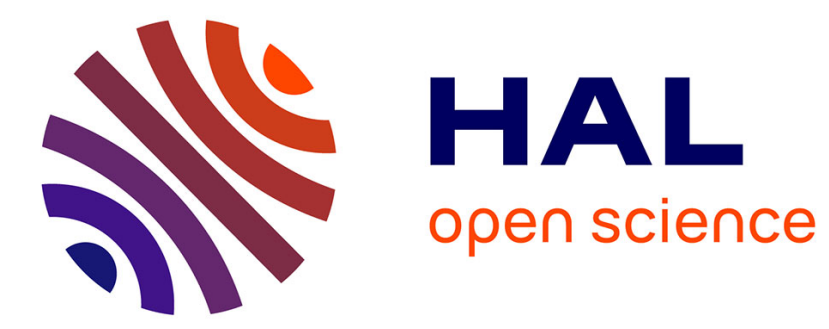

\title{
Malaria control and infant mortality in Africa
}

Denis Cogneau, Pauline Rossi

\section{- To cite this version:}

| Denis Cogneau, Pauline Rossi. Malaria control and infant mortality in Africa. 2019. hal-01543033v2

\section{HAL Id: hal-01543033 \\ https://hal-pjse.archives-ouvertes.fr/hal-01543033v2}

Preprint submitted on 26 Sep 2019

HAL is a multi-disciplinary open access archive for the deposit and dissemination of scientific research documents, whether they are published or not. The documents may come from teaching and research institutions in France or abroad, or from public or private research centers.
L'archive ouverte pluridisciplinaire HAL, est destinée au dépôt et à la diffusion de documents scientifiques de niveau recherche, publiés ou non, émanant des établissements d'enseignement et de recherche français ou étrangers, des laboratoires publics ou privés. 


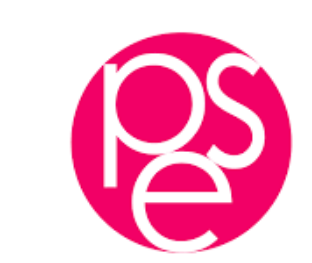

PARISSCHOOL OFECONOMICS
ECOLED'ECONOMIEDE PARIS

WORKING PAPER N 2017 - 02

Malaria Control and Infant Mortality in Africa

Denis Cogneau

Pauline Rossi

JEL Codes: I1, J1, 01, F35

Keywords: Child mortality, Malaria, Africa, Foreign aid 


\title{
Malaria Control and Infant Mortality in Africa
}

\author{
Denis Cogneau and Pauline Rossi*
}

August 2019

\begin{abstract}
Has massive distribution of insecticide-treated-nets contributed to the reduction in infant mortality in Sub-Saharan Africa over the past 15 years? Using large household surveys collected in 16 countries and exploiting the spatial correlation in distribution campaigns, we estimate the relationship between the diffusion of bednets and the progress in child survival. We find no evidence of a causal link in cities, and no impact either in rural areas with low malaria prevalence. By contrast, in highly malarious rural areas where bednet coverage reached high levels, above $75 \%$ of households, infant mortality has been reduced by at least 3 percentage points, which amounts to $25 \%$ of the initial mortality. The identified impact is even higher for the children of mothers with no education. It lies at the upper bound found with RCTs, most likely because those were implemented in contexts with lower mortality and/or malaria prevalence.
\end{abstract}

Keywords: Child mortality, Malaria, Africa, Foreign aid. JEL Codes: I1, J1, O1, F35.

*Denis Cogneau (corresponding author): Paris School of Economics, IRD, EHESS, 48 boulevard Jourdan 75014 Paris, denis.cogneau@psemail.eu. Pauline Rossi: University of Amsterdam, CEPR and Tinbergen Institute. Roeterstraat 11, 1018WB Amsterdam. The authors are grateful to Kenneth Houngbedji for his help in data construction. They also thank Pascaline Dupas, Sylvie Lambert, François Libois and Karen Macours for helpful comments, and seminar or conference participants at CREST, DIAL/Paris-Dauphine, Oxford University and PSE. The usual disclaimer applies. 


\section{Introduction}

One of the big success stories of the global fight against poverty is the substantial reduction in child mortality experienced worldwide in recent years. According to the World Development Indicators, the infant mortality rate was halved between 1990 and 2015, from 6.5\% to 3.1\%, and there was an acceleration of the progress in the last 10 years. In this global endeavor, SubSaharan Africa has played and will play a crucial part, because it is the world region with the largest room for improvement. It is the region where the decrease was strongest in absolute terms, from $10.8 \%$ in 1990 down to $5.5 \%$ in 2015. There has been substantial progress, but the mortality rate is still almost twice as large as the world average. As a result, half of child deaths in 2015 occurred in Africa (UN Inter-agency Group for Child Mortality Estimation, 2015). In the future, this share is predicted to increase because the population growth is much larger in Africa than anywhere else in the world. Indeed, an estimated $40 \%$ of births will take place in Africa in 2050. Child mortality will thus increasingly become an African issue (UNICEF, 2014). Together with immunization campaigns, one large-scale health intervention is usually cited as a key success factor: the fight against malaria, coordinated by the publicprivate partnership Roll Back Malaria (United Nations, 2015).

Malaria has been eradicated from various parts of the world during the twentieth century. Today, the burden weighs mostly on Sub-Saharan Africa, where we find the largest prevalence rates and the most dangerous form of malaria. At the beginning of the twenty-first century, there was a series of initiatives launched by the international community to start the fight against malaria in Africa. Very large-scale interventions have been implemented, among which the most emblematic is the distribution of Insecticide-Treated Nets (henceforth ITNs). 900 millions of bednets have been distributed since the early 2000s. Nowadays, an estimated $2 / 3$ of children sleep under an ITN against virtually none before the distribution started (WHO, 2015). Another type of intervention is improving access to curative treatments called 
Artemisinin-based Combination Therapy. The scope of this intervention is more modest with only an estimated $16 \%$ of children being treated when they are sick in 2015 . The last type of intervention is to have public agents spray the inside of dwellings with an insecticide (Indoor Residual Spraying). This type of intervention historically eradicated malaria in many places. Nowadays, because of undesirable side effects it is less promoted and covers less than $5 \%$ of the population at risk in Africa. Since the start of Roll Back Malaria, the evolution of the disease in terms of prevalence and mortality has been closely monitored by the WHO. According to their estimates, the prevalence among children decreased from $33 \%$ in 2000 down to $16 \%$ in 2015 , and the number of deaths caused by malaria among children under 5 years old decreased from $700 \mathrm{~K}$ per year down to 300K (WHO, 2015). The Millenium Development Goals Report 2015 concludes that the "substantial expansion of anti-malaria efforts have helped [...] improve child survival rates" (United Nations, 2015).

The goal of this paper is to challenge these statistics and assertions. Have malaria control efforts really contributed to the reduction in infant mortality in Sub-Saharan Africa over the past 15 years? Or have they simply coincided with favorable time trends? If they did make a difference, by how much and for whom? We face two main empirical challenges to answer these questions. First, measuring properly the progress in malaria control in countries with weak public health systems and no vital statistics; second, identifying a causal relationship when the intervention was not randomly allocated.

We believe that it is worth trying to overcome these issues because the question is of primary relevance for policy-makers. The Roll-Back Malaria initiative is a very large-scale, costly program. 2.5 billion dollars were spent in 2015, of which 1.5 went to Sub-Saharan Africa. The cumulated costs since 2000 exceed 20 billion dollars. As a point of comparison, approximately 5.5 billion dollars are spent each year by the international community on food aid. ${ }^{1}$ Anti-malaria efforts are mostly funded by international organizations, such as the

\footnotetext{
${ }^{1}$ Data from the World Food Program http://www.wfp.org/funding/year/2014
} 
Global Fund to fight AIDS, tuberculosis and malaria, as well as development aid agencies of developed countries, the main contributors being the US and the UK. In many African countries, the program is in fact entirely funded by international aid (WHO, 2015). This paper contributes to the lively debate on the efficiency of aid by analyzing if a prominent global health intervention yielded some returns, and for whom.

The answer is not obvious ex-ante. In spite of the enthusiasm of international organizations, the efficiency of the program has been questioned. Some have pointed to anecdotal cases where nets are used for fishing or for soccer. However it seems that many households having received a free net actually use it. This is found in RCTs like the ones of Hoffmann (2010) in Uganda or of Dupas (2014) in Kenya. In Kenya again, Hightower et al. (2010) find that having received a campaign bednet greatly increased the likelihood of a child under 5 slept under an ITN the previous night. In our surveys sample, among households owning a bednet and having under 5 children, two thirds declare that some of the children slept under a net during the previous night, both in urban and rural areas. The question is rather whether the free distribution of ITNs is sufficient to significantly impact infant mortality, and to which extent, as there is quite a long chain from distribution to sustained (individual and collective) usage, and finally to mortality reduction. Experimental results from the medical literature show that ITNs do reduce infant mortality "under highly controlled conditions, leading to high coverage and use rates" (Lengeler, 2004, p.10). Whether these conditions hold for at-scale interventions is an open question of interest. Over 150 local longitudinal or cross-sectional studies have been published in recent years by medical journals to assess the effectiveness of control efforts on malaria prevalence, and $38 \%$ find no significant results (Kesteman et al., 2017). Among those few studies that looked at infant mortality as well, both zero, small and large effects were found. We contribute to this literature by improving the methodology in terms of external and internal validity.

In this paper, we examine the relationship between the distribution of bednets and the 
evolution of infant mortality (0-12 months old). ${ }^{2}$ We exploit the spatial dimension of campaigns to measure the treatment, i.e. the distribution of bednets, at a very disaggregated level. We show that changes in bednet coverage are spatially correlated and that information in the neighborhood can be used to compensate for missing data in a given geographical unit in a given year. Thus, we are able to construct a direct, data-based measure of the treatment across time. We find that infant mortality did decrease more where more bednets were distributed, but only in rural areas with high initial malaria prevalence; no causal impact is found in urban areas or in lowly malarious rural areas. In highly malarious rural areas, we estimate that the surge in bednet ownership during the period is associated with a reduction in infant mortality by at least 3 percentage points, equivalent to $25 \%$ of baseline infant mortality, in places where bednet coverage reached a high level (above $75 \%$ ). In terms of magnitude, our correlation lies at the upper bound of experimental results from the medical literature: according to a Cochrane review of randomized control trials (henceforth RCTs), ITNs reduce child mortality by 14-29\% compared to no nets (Lengeler, 2004; Richardson et al., 2018).

Unlike most existing articles, we are able to make strong claims about external validity because we exploit nationally representative surveys (the Demographic and Health Surveys) in 16 countries, covering around one half of the population in Sub-Saharan Africa. ${ }^{3}$ In terms of internal validity, we discuss the fact that our correlation potentially captures a bunching of health interventions, a change in population characteristics, and an endogenous placement of ITNs. Yet, our estimates remain stable after controlling for vaccination coverage and prenatal care supply, luminosity, mother fixed effects, and do not pick up pre-trends. Causal inference seems in particular most credible for the survival of children of uneducated mothers living

\footnotetext{
${ }^{2}$ We of course acknowledge that malaria control may have other impacts, on morbidity and productivity in particular.

${ }^{3}$ Another advantage of our database is the large number of observations, which gives us the high statistical power needed to examine relatively rare events like child deaths. This is crucial because the two-stage sample design of surveys and our reweighing of country samples both limit the level of precision that can be achieved.
} 
in the malarious rural areas, as migration bias are limited in this case; for these children, we even get a higher impact of $4 \mathrm{pp}$, equivalent to $30 \%$ of their baseline mortality. Overall, we make a fairly positive assessment of the distribution of ITNs. They have contributed to substantially improve child survival in disadvantaged populations.

The rest of the paper is organized as follows. Section 2 makes a brief review of the medical and economic literature on the impact of ITNs. Section 3 discusses data issues. Section 4 examines preliminary evidence at country level and at sub-national level. Section 5 presents our analytical methodology. Section 6 discusses our main results. Section 7 concludes.

\section{Literature}

This paper relates both to the medical literature and the economics literature.

\subsection{Medical literature}

The medical literature is primarily interested in assessing the efficacy of curative or preventive treatments against malaria on child mortality using RCTs. Researchers further perform some meta-analysis to come up with a global estimate of the treatment effect. Lengeler (2004) reviews the results of $5 \mathrm{RCTs}$ estimating the impact of ITNs on under-5 mortality in Africa. Estimates range from $-14 \%$ to $-29 \%$. These studies are very helpful to demonstrate the impact of treatments in "ideal" conditions but they are not necessarily informative about the progress to be expected in real-life conditions, for two reasons. First, special attention is paid in delivering the intervention during controlled trials and it is unclear whether such conditions can be fulfilled at a larger scale. If they cannot, experimental estimates are too optimistic. Second, trials are conducted in a limited number of settings that are not

representative of all field conditions. 3 out of the 5 RCTs were conducted in Kenya and the Gambia, where malaria is not endemic. If bednets save more lives where more children 
initially die from malaria, these coefficients underestimate the impact of distributing ITNs in endemic areas. Moreover, the under-5 mortality rates are extremely low in the 5 settings considered: between $1.3 \%$ and $5.2 \%$ in the control groups against $15 \%$ on average in SubSaharan Africa when the RCTs took place (in the 1990s). Again, if the treatment effect depends on initial mortality rates, RCTs estimates are not informative about the average population.

Researchers have therefore also studied the effectiveness of malaria control interventions, in particular the impact of ITN distributions in real-life, at scale conditions. A meta-analysis by Kesteman et al. (2017) provides a recent systematic overview of this literature. They document a surge in the number of publications since 2009, reflecting the growing general interest in this question. The vast majority of articles are either local longitudinal studies, comparing outcomes before and after the campaigns, or cross-sectional studies, comparing areas/individuals with and without ITNs. They have important limitations in terms of external validity $(2 / 3$ are at the sub-national level) and internal validity (for instance $1 / 5$ do not control for any covariate). There are two outcomes of interest: parasitemia and number of acute cases. $62 \%$ of studies find a significant negative correlation with ITN use or ownership, while $38 \%$ find no significant result. A handful of articles look at child deaths and report negative coefficients with very large standard errors, covering the range [0;75\%] reduction in mortality. Only two studies have large, multinational samples, and try to isolate a causal effect using statistical models. Lim et al. (2011) exploit DHS in 22 African countries to estimate a cross-sectional matching model. ${ }^{4}$ They find that under-5 mortality is significantly lower by $23 \%$ in households who own an ITN. Using a similar method, Eisele et al. (2012) find no significant relationship with neonatal mortality.

To critically assess the orders of magnitude found in the literature, quantifying the burden

\footnotetext{
${ }^{4}$ The matching is coarse, with four variables: child age, mother education, rural dummy, malaria transmission zone $=$ high, medium or low.
} 
of malaria on child deaths is helpful. This is a challenging task since there is no national vital registration systems categorizing all deaths by cause in African countries. The secondbest source of data are verbal autopsy reports, in which the caregiver (sometimes a health worker, often a family member) is asked about the cause of the death. These reports provide information for some locations at some points in time. Next, researchers use statistical methods to extrapolate the results to larger units, like the continent. In this way, Black et al. (2014) estimate that $16 \%$ of under-5 deaths in the whole continent were caused by malaria in 2008. Control efforts can therefore not reduce child mortality in a representative sample of Africa by much more than that.

In this article, we contribute to evaluating the effectiveness of recent malaria control interventions by improving the methodology in terms of external and internal validity. Kesteman et al. (2017) conclude their literature review by the following recommendations for future research: "sample all geographical and/or cultural patterns, stratify the evaluation [...], adjust for socio-demographic variables that are associated with the outcomes". In a special issue of the American Journal of Tropical Medicine and Hygiene dedicated to this topic, Yazoume et al. (2017) come to the same conclusion. In their expanded framework for evaluating the health impact of the scale-up of malaria control interventions on all-cause child mortality in Sub-Saharan Africa, they encourage researchers to pay specific attention to heterogeneous effects and to combine spatial and temporal variations. This is precisely what we try to do.

\subsection{Economics literature}

As far as economists are concerned, they have been mostly interested in identifying the causal impact of malaria on socio-economic outcomes, like income or education. ${ }^{5}$ This strand of

\footnotetext{
${ }^{5}$ Others have investigated determinants of ITN adoption and use. Dupas (2009), Cohen and Dupas (2010), and Dupas (2014) estimated price-elasticities in Kenya, while Tarozzi et al. (2014) studied the uptake of ITNs through micro-loans in Orissa (India), and found disappointing evidence for the impact on malaria prevalence and on health, that they attribute to low adoption.
} 
the economics literature fits into a global debate about the relationship between disease and development. Three papers set the golden rule for identification: Bleakley (2010) in the US, Brazil, Colombia and Mexico; Cutler et al. (2010) in India; Lucas (2010) in Paraguay and Sri Lanka. They all use eradication campaigns as natural experiments, exploiting the variation in initial prevalence of malaria. The idea is to perform a difference-in-difference before and after the campaign, comparing areas with high and low initial prevalence. The assumption is that the eradication campaign caused a bigger change in areas more affected by malaria. The general message of these papers is that exposure to malaria in childhood deteriorates human capital. Note that the three papers abstract from child mortality, which might in principle raise an issue of selection. However, they study countries in America and South Asia, where malaria causes milder symptoms, rarely death, so the scope for such a concern is limited.

Following these seminal contributions, several papers have exploited the same strategy to study e.g. cognitive skills in Mexico (Venkataramani, 2010) and anemia in Africa (Apouey et al., 2017). Importantly for us, two recent working papers examine the impact on infant mortality and fertility in Africa (Kuecken et al., 2017; Wilde et al., 2017). Both papers exploit various waves of DHS in several African countries together with data from the Malaria Atlas Project (Bhatt et al., 2015). The Malaria Atlas is a map constructed by epidemiologists showing estimated malaria prevalence and ITN use at a fine geographical level for each year between 2000 and 2015. It is possible to merge the Malaria Atlas with DHS to get a proxy for the disease environment in which each child was born. The authors interact the initial prevalence of malaria with a measure of campaigns intensity to estimate a causal impact under some parallel trend assumptions. They come to different conclusions: Wilde et al. (2017) find no significant impact on infant mortality and a positive impact on fertility, while Kuecken et al. (2017) find very large negative impacts on both dimensions, in particular a 5 pp (roughly 50\%) reduction in infant mortality. 
The main empirical challenge is that malaria has not been eradicated yet, so the initial prevalence is not mechanically related to the intensity of the change. Comparing high and low prevalence areas is therefore not enough. A measure of the treatment is needed, i.e. observing the distribution of bednets, and this is not straightforward. Two options have been pursued so far: an indirect, country-wide measure of control efforts like money spent by international donors (Kuecken et al., 2017), and an estimate of ITN use predicted by epidemiological models like Malaria Atlas (Wilde et al., 2017); actually the two options are quite close because the time variation of ITN use from the Malaria Atlas (Bhatt et al., 2015) is anchored on the evolution of the available stock of bednets in the country (see the data section below). Very recently Dolan et al. (2019) use DHS data from the Democratic Republic of Congo and also implement a double-difference strategy based on the dates of the bednets distribution campaigns in each province; like the other references and in contrast with us, they do not make use of the information on bednets ownership at the local level. We choose a more direct data-based approach exploiting the spatial dimension of the distributions. ${ }^{6}$

\section{Data}

Ideally, we would like to have time series to monitor the evolution of the outcomes, infant and child mortality rates, and the evolution of malaria control efforts, both in terms of outputs like the malaria prevalence among children, and in terms of inputs like the coverage of interventions. We would like to observe these quantities at the finest geographical level and for the period of interest between 2000 and 2015, as well as the decade before to look at pre-trends. If these time series existed, estimating the correlation between malaria control

\footnotetext{
${ }^{6}$ A last study by Kudamatsu et al. (2012) is close to our paper in the sense that authors are interested in the relationship between malaria and infant mortality in Africa. But their perspective is quite different from ours. Their goal is to identify the causal impact of weather shocks on infant mortality. The motivation is to better understand the health consequences of climate change. For them, malaria is a channel through which unusually hot and humid weather conditions may cause extra deaths. They find that non-malarious weather conditions in utero reduce infant mortality by 0.8 p.p.
} 
efforts and the change in infant mortality would be quite straightforward.

The Demographic and Health Surveys (DHS) conducted in African countries between 2000 and 2015 contain part of the information needed. ${ }^{7}$ In particular, we can reconstruct the evolution of mortality from the retrospective information on birth histories of mothers, including date of birth, survival status and, if applicable, date of death. However, the information on malaria control is limited. We have no data on malaria prevalence, and no retrospective information on bednets. We know if the household owns and uses a bednet at the time of the survey, but not since when. So we cannot infer which child has been protected by a bednet since he/she was born. To be more specific, we know (i) if the households own a bednet, and if they do, (ii) if children under 5 slept under a bednet the night before and whether these bednets were treated or untreated. The latter information is not recorded in all early survey waves. In our analysis, we rely on the ownership variable because we want to assess the impact on the whole population, not only on bednet-users, so we are interested in an intent-to-treat estimator. Moreover, bednet ownership seems more reliable: it can be observed by the enumerator and it is less prone to recall or declaration bias. The type of bednet owned is not recorded in all early survey waves either. We then measure bednet coverage, whether treated or untreated, and will have non-zero bednet coverage before the massive distribution of ITNs by the international campaign. Yet, after 2002 the bulk of the increase in bednet coverage was due to the campaign, so that all the variation that we exploit corresponds to treated nets.

Our strategy to reconstruct the evolution of the intervention coverage is to exploit the spatial dimension. As explained in the next section, we need at least two waves of DHS in the same country since the early 2000s, when the distribution of bednets started. We also

\footnotetext{
${ }^{7}$ DHS provide stratified samples of mothers aged 15 to 49 . Individual survey weights ensure that the survey sample is representative of all mothers at the country level. Nonetheless, sample size of surveys is not proportional to population size. To obtain a representative sample of the countries studied, we reweigh the whole sample. This results in a lower efficiency of estimates. We test the robustness of our results to this reweighing.
} 
need the GPS coordinates of the geographical cluster where households are located. Among available countries, we selected out Ethiopia, Namibia and Zimbabwe where malaria is not widespread. In the end, there are 16 countries with geocoded data in at least 2 waves of DHS over the relevant period. The corresponding surveys are listed in Table A.1 in Appendix. Kenya, Rwanda and Senegal are the three countries in our sample where the prevalence of malaria is the lowest (see Figure A.1 in Online Appendix). ${ }^{8}$

As explained in the literature review, an alternative measure of malaria control efforts is provided by the Malaria Atlas. We explain here why we chose not to rely on it. We refer to the large supplemental appendix of Bhatt et al. (2015). Bayesian modelling is used in order to fit a joint statistical model of malaria prevalence and anti-malaria interventions (ITN distribution, indoor residual spraying and artemisin based combination therapies) at a 2.5 Arcminute $(\sim 5 \mathrm{~km} \times 5 \mathrm{~km})$ spatial resolution and across the $2000-2015$ period. Data come from the DHS programme surveys and a few other nationwide household surveys of the same kind, which provide blood testing on malaria prevalence, and ITN ownership and use indicators like the ones we use. A large set of environmental covariates from remote sensing databases like MODIS is used to predict base malaria prevalence in 2000 (Online Figure A.1). We will use this estimate of initial malaria prevalence to distinguish lowly malarious areas from highly malarious areas.

Then national malaria control programs data were used to calibrate the average availability of ITNs in each country at a given year, then sub-national ITN access was predicted using environmental variables not used in the prevalence model, and ITN use was modelled on top of ITN access adding non-parametric seasonal trends and a Markov field of unobserved spatio-temporal random effects. For other interventions, only national-level data are available. Then for years after 2000, the malaria prevalence model includes spatio-temporal

\footnotetext{
${ }^{8}$ DHS waves implemented before 2000 (DHS-III or lower) did not record bednets ownership and hence can not be used.
} 
variations in environmental conditions, ITN use and other interventions, along with countryspecific random effects and again a Markov field of unobserved structural random effects. This means that the model proposes an evaluation of the impact of ITN distribution campaigns on malaria prevalence, and the authors indeed conclude they were very effective in reducing the Plasmodium Falciparum infection prevalence by $34 \%$, hence avoiding a lot of clinical cases.

The ITN use variable of the Malaria Atlas is thus an unknown (to us) function of environmental characteristics explaining bednets ownership and use, and yearly national-level distribution campaign data. In its spirit, it looks very much like a Bartik instrument of actual (unobserved) use in each location (Goldsmith-Pinkham et al., 2018). This function being non-linear, due to a logit specification, environmental characteristics should not cancel out with cluster fixed effects, and could then act as confounding factors for the impact of ITN access or use on mortality. This "black box" characteristic of the Atlas variable makes us prefer to build up a more transparent approach. ${ }^{9}$

\section{Preliminary evidence on the correlation of interest}

Before describing our measure of malaria control efforts, we provide some descriptive evidence of the correlation between bednets and infant mortality.

\subsection{Cross-sectional analysis}

We start by estimating the correlation between bednet ownership/use and infant mortality in a cross-section of households using the latest wave of DHS in each country. In columns (1) and (2) of Table A.2 in Appendix, we perform an analysis between clusters, regressing the infant mortality rate in a given geographical cluster (primary sample unit of DHS) on

\footnotetext{
${ }^{9}$ Furthermore, our main strategy use bednets coverage in a $10 \mathrm{~km}$ radius around DHS clusters, so that the spatial resolution of this ITN use variable is not higher than ours.
} 
the proportion of households owning/using a bednet in the same cluster. Both for urban and rural clusters, we find a positive relationship: children die more in areas with a high coverage of bednets. This is because bednets are not needed in malaria-free areas. To assess their impact on child survival, it is essential to control for the riskiness of the environment.

This is what we do in columns (3) and (4) of Table A.2 by performing an analysis within cluster. We compare the mortality rates of children born the same year in the same cluster - so they are facing the same environment - some owning/using a bednet while others do not. The correlation is now negative, and very significant for bednet use (bottom panel): in rural areas those sleeping under a bednet are 1 pp less likely to die. However, households using a bednet are probably different from the others, and these specific characteristics might explain the survival advantage. We control for mother's age, education and wealth but there remains some unobserved heterogeneity such as mother's care for the children that we cannot account for using a cross-section. On the other hand, these estimates disregard within-cluster externalities: if what matters is not so much individual use but the number of neighboring households who use, the impact of bednet coverage could be underestimated. This could explain why the coefficients for bednet ownership (top panel) are small and insignificant.

\subsection{Panel analysis: country-level}

We therefore turn to panel analysis. At the country level, we have data on mortality rates and bednet ownership/use at two points in time. ${ }^{10}$ Figure 1 plots, for each country in our sample, the variation in infant mortality on the $y$ axis, and the variation in bednet coverage on the $x$ axis. Infant mortality is measured on children born 1 to 4 years before surveys date, and bednet coverage is measured as the share of 15-49 year-old mothers having at least one bednet at home. When we consider all areas together, the relationship is negative: countries

\footnotetext{
${ }^{10}$ For countries with 3 waves of DHS during the period 2000-2015, we here consider the first and third waves to have the longest time span.
} 
with a stronger take-up in bednets also have a faster decline in mortality. By fitting a linear regression on the raw data, we get a negative although quite imprecise coefficient equal to -0.036 with a standard error of 0.023 . When we consider rural and urban areas separately, we uncover substantial heterogeneity. In rural areas, there is a stronger negative correlation between mortality and bednets, with a coefficient of -0.054 and a standard error of 0.026 . On the contrary, the relationship in urban areas is, if anything, positive.

The main shortcoming of country-level analysis is that there are many variables driving both the evolution of mortality rates and the distribution of bednets. They may either underestimate or overestimate the impact of bednets on mortality. For instance, in countries with a poor health infrastructure, the distribution of bednets might be slower as well as the progress in child survival. On the contrary, if international donors target specifically those countries in which public health is quickly deteriorating, a surge in bednets might coincide with a worsening of survival indicators. To get rid of variables determined at the country level, it is necessary to consider a finer geographical level.

\subsection{Panel analysis: sub-national level}

One first possibility is superimpose a grid of squares on the map of countries, and correlate the variation in infant mortality with the variation in average bednet coverage in each square; country fixed effects can cancel out the country-level confounders mentioned above. We do this in Table 1, for three levels of resolution : $0.5 \times 0.5,0.25 \times 0.25$ and $0.1 \times 0.1$ degree squares. As many samples of squares can be constructed depending on how we position the grid, we provide the average of the estimates from 25 different grids (see Table footnote for details). Through this procedure, we inevitably do not cover all areas in each country, as seen when comparing the theoretical number of squares with the number of squares with non-missing data; with the intermediate definition of $0.25 \times 0.25$, one half of the squares are missing. Some squares are so sparsely populated that they do not contain a DHS cluster in 
both waves. This sample selection of course increases with the grid resolution. On the other hand, the $0.5 \times 0.5$ grid is quite coarse (at the Equator, 0.5 degrees is around 50 kilometers). Note that we still measure infant mortality on children born 1-4 years before survey date, so that internal migration should not be a large source of bias.

Although estimates are very imprecise, we still find a negative correlation between infant mortality and bednet coverage for the whole sample and for rural areas. Like at country-level, the correlation is rather positive when restricting to urban areas. The negative correlation reaches $95 \%$ significance for the rural sample and the intermediate definition of $0.25 \times 0.25$ degrees: -0.023 with a standard error of 0.011 . The rural negative correlation increases in absolute value with the definition of the grid, from -0.016 to -0.033 , while the positive correlation decreases in urban areas from +0.041 to +0.026 .

The same procedure allows us to measure the spatial correlation in bednets diffusion. The two maps of Figure 2 depict the evolution of the spatial distribution of bednet coverage across time, at the $0.5 \times 0.5$ degree definition. In the first DHS waves (left map), ranging from 2000 to 2008, only a few squares, mainly in Mali and Zambia, have a coverage lying above $75 \%$. The earliest survey for Zambia is from 2007; yet the campaign does not seem to have reached DRC, Liberia and Sierra Leone which were also surveyed after 2007. In the last waves, ranging from 2010 to 2014, the progress in coverage is very impressive, with Cameroon 2011 standing as one major exception. In countries like Burkina Faso, DRC, Liberia, Nigeria, Sierra Leone and Zambia, the regional distribution of bednets is still uneven. Table 2 looks at the spatial correlation in the variation of coverage, by regressing the variation in one square on the average variation of the eight (potential) neighboring squares. Like with the previous analysis, we again vary the resolution of the grid. The effect of resolution on sample selection is even stronger than in the previous analysis, as we additionally need to have non-missing data for at least one neighbor. For rural areas, the variation in coverage is quite spatially correlated, as the point estimates of the coefficients range between 0.38 and 0.70 ; yet the 
highest resolution results in analyzing only $10 \%$ of the squares, so that we believe that lower resolution estimates are more reliable $(0.58$ for $0.5 \times 0.5$ and 0.70 for $0.25 \times 0.25)$. For urban areas, the estimates are lower, ranging from 0.18 to 0.39 ; however, the analysis on urban areas is highly selected and biased, even with the lowest resolutions, as many cities do not have another urban neighbor in a 10 to 50 kilometers radius. If the city is large enough, multiple blocks in the city will be surveyed in each wave, yet will lie within the same square.

An alternative solution for the sub-national analysis is to go down to individual survey clusters defined in DHS, covering a village or a block in a city, and use the nearest neighbors of these clusters across survey waves in order to measure the distribution of bednets in their vicinity. Of course, this will only be possible for a selected sub-sample of clusters that have a close enough neighbor in each survey wave; like in the grid analysis, external validity will be limited by this sample restriction. At the same time, we will hopefully gain precision. And, by making use of the retrospective data on child birth and child mortality, we will also progress in internal validity.

\section{A spatial analysis of the variation in ITN access}

\subsection{Main estimation strategy}

As explained in section 3, there is no indicator measuring the evolution of bednet access at the cluster level. For each DHS wave, we observe the proportion of households owning a bednet at the date of the survey in the clusters of the survey. However, sampling units are randomly drawn at each survey. So clusters drawn in the first wave are generally not surveyed in the second wave. Yet, other clusters in the neighborhood are. They provide information on the intensity of anti-malaria campaigns in the area. Our idea is to exploit the spatial correlation in the distribution of bednets, using the geographic coordinates of clusters. 
For each survey cluster $c$ in country $j$, let us denote $n\left(c, w_{j}\right)$ its closest neighbor in survey year $w_{j}$. For any birthyear $t$, we define $B_{n(c, t)}=\bar{B}_{n\left(c, w^{*}\right)}$ as the average ownership of bednets by households in neighboring $n\left(c, w^{*}\right)$, where $w^{*}=\underset{w_{j}}{\arg \min }\left|t-w_{j}\right|$, i.e. the closest survey date to $t$. We drop children whose birth year is more than 5 years before the first survey date $\left(\left|t-\min \left(w_{j}\right)\right| \geq 5\right)$; we do not interpolate coverage rates between survey waves, in order to keep with a data-based approach. We hypothesize that the mortality risk of a child $i$ born to mother $m$ in cluster $c$ at year $t$ is impacted by $B_{n(c, t)}$, either "directly" through bednets prevalence in $c, B_{c t}$ - that we do not observe except around the survey wave to which $c$ belongs, or "indirectly" through bednets and hence mosquito prevalence in the area. Our basic (linear) specification is the following:

$$
y_{i c m j t}=\alpha B_{n(c, t)}+\beta_{1} X_{1 i}+\beta_{2} X_{2 m}+\gamma_{j t}+\delta_{c}+\zeta Z_{n(c, t)}+\epsilon_{i m c j t}
$$

$y_{i c m j t}$ is a dummy variable indicating whether child $i$ died after one year (infant mortality) or one month (neonatal). $\gamma_{j t}$ is a birthyear-country fixed effect, capturing all determinants of infant mortality specific to a birth cohort in country $j . \delta_{c}$ is a cluster-area fixed effect, accounting for all time-invariant characteristics related to the survival of children in cluster $c$. $X_{1 i}$ is a vector of controls including child-specific variables (sex and mother's age at birth) and $X_{2 m}$ a vector of mother or household-level characteristics (wealth index, education of mother and husband, access to electricity, radio ownership). $Z_{n(c, t)}$ is a vector of characteristics of cluster $c$ neighbor containing variables that may confound the impact of $B_{n(c, t)}$ : averages of $X$ controls in $n(c, t)$, and most importantly proxies for other health interventions that might have been bunched with bednet distribution: vaccination or prenatal care. Errors $\epsilon_{i m c j t}$ are clustered at survey cluster level $c$; we also tried clustering at the level of $n\left(c, \min w_{j}\right)$, for earlier surveys usually have smaller sample sizes so that it is often that two clusters share the same neighbor. $\alpha$ is a kind of intent-to-treat coefficient: we seek to estimate the impact on 
infant mortality of distributing bednets to households in an area, without caring for actual use. The neighborhood coverage rate $B_{n(c, t)}$ might be also transformed in a categorical variable, in order to allow for a non-linear impact of the bednets treatment.

We estimate equation (1) on the pooled sample of children born to 15-49 year-old mothers interviewed in each country's last survey wave $\max \left(w_{j}\right)$, so that children born in the most recent years were potentially exposed to bednets distribution campaigns, whereas children born earlier were not. We temporarily disregard migration and assume that $B_{n(c, t)}$ is a good proxy of the prevalence of bednets in the neighborhood, for children observed in cluster $c$ whatever their birthyear $t$. For old enough mothers having given birth to more than one child, an alternative specification replaces the cluster-area fixed effect $\delta_{c}$ by a mother fixed effect $\theta_{m}$, absorbing all time-invariant characteristics of mothers and households:

$$
y_{i m c j t}=\alpha B_{n(c, t)}+\beta_{1} X_{1 i}+\gamma_{j t}+\theta_{m}+\zeta Z_{n(c, t)}+\epsilon_{i m c j t}
$$

A variant of equations (1) or (2) also includes a term $\lambda_{c} t$, i.e. a cluster-area linear time trend, making identification of $\alpha$ rely on non-linear variations of $B_{n(c, t)}$ across time, obtained from countries with three waves of DHS.

Our strategy relies on variable $B_{n(c, t)}$ capturing correctly the bednets environment of the child, in particular on observed increases in bednet prevalence reflecting effective improvements in coverage and not a spurious increase in the accuracy of measurement. With this aim in view, we use neighbor's prevalence even in the case where we do observe $B_{c t}=\bar{B}_{c w^{*}}$, for children born recently in the last survey wave, i.e. such as $w^{*}=\max w_{j}$. As the preliminary evidence of the previous section has suggested that urban and rural areas might be differently impacted, we match urban clusters with their nearest urban neighbors, and rural clusters with their nearest rural neighbors, and we conduct the estimation separately on 
the two kinds of clusters. ${ }^{11}$ We also restrict the estimation sample to survey clusters whose nearest neighbor is no further than $10 \mathrm{~km}$ in each wave. ${ }^{12}$ In DHS data, GPS coordinates are randomly displaced by a maximum of 5 kilometers for rural clusters ( $2 \mathrm{~km}$ for urban), with even $1 \%$ of rural clusters being moved up to $10 \mathrm{~km}$, so that looking for even closer neighbors would be meaningless. We tried redefining $B_{n(c, t)}$ as the average bednets prevalence in a $10 \mathrm{~km}$ radius, i.e. potentially averaging over multiple neighbors, however this made little difference.

More formally, we hope to indirectly measure the impact of $B_{c t}$ that we only partially observe, if it is sufficiently correlated to $B_{n(c, t)}$ :

$$
B_{c j t}=\rho B_{n(c, t)}+\mu_{j t}+\nu_{c}+\eta_{c j t}
$$

With some knowledge of $\rho$, the effect of $B_{c t}$ on $y_{i c m j t}$ could then be computed as $\alpha / \rho$ (if we assume that all the impact of $B_{n(c, t)}$ goes through $\left.B_{c t}\right)$. The grid squares analysis in the previous section suggests that for rural areas $\rho$ could lie above 0.7 , as we expect the spatial correlation of bednet diffusion to be higher with close neighbors (by less than $10 \mathrm{~km}$ ) than with large grid squares; this means that the true effect of $B_{c t}$ could be underestimated by at most $40 \%$ ( $\rho \approx 0.7)$. As explained above, in the case of urban areas the inter-temporal spatial correlation is harder to estimate with grid squares.

The main drawback of this "neighbor strategy" is that we cannot exploit half of the clusters, because they lack a close neighbor in previous waves. We are able to keep $2 / 3$ of the clusters in urban areas and $1 / 3$ in rural areas. Selected clusters are are more often from countries of small surface area (Benin rather than DRC) and/or where sample sizes are higher

\footnotetext{
${ }^{11}$ Another line of heterogeneity that we explore is education of mother, that additionally allows us to discuss the impact of selective migration.

${ }^{12}$ More precisely, for clusters which have no close enough neighbor in the intermediate survey wave but in one of the two extreme waves, we disregard the intermediate measurement of $B_{n(c, t)}$ and match children to $\bar{B}_{n\left(c, \min w_{j}\right)}$ or $\bar{B}_{n\left(c, \max w_{j}\right)}$ depending on their birthyear.
} 
(like Kenya). Tables A.3 and A.4 in the Online Appendix provide detailed information on the sample selection. There is no difference as for malaria prevalence and infant mortality in rural areas. But generally, selected clusters are better off in terms of wealth, education and access to health care; they are also closer to the biggest city in the country, and selected urban areas are more often found in larger cities that are more likely to be surveyed in each wave. Thus it should kept in mind that our results hold for less peripheral areas; we lack information on the neighborhood of small cities or villages that are too isolated. We discuss more extensively in the last section how these results could be extrapolated to other contexts.

\section{$5.2 \quad$ A quasi-experimental setting}

In the specification that we have just set, bednets coverage in the environment of the child is a continuous treatment whose exogeneity to mortality determinants might still be questioned. To go further, we build up a more transparent difference-in-difference framework distinguishing treated and control areas with a discrete treatment. We exploit the fact that high rates of bednets coverage seem to make a large difference in terms of mortality and define treatment as reaching the threshold of $75 \%$ mothers with a bednet at home. As seen in Figure 2, more than $90 \%$ of survey clusters had coverage rates much below the $75 \%$ threshold before the mid-2000s, while by the end of the decade half of them had reached the threshold. So we can estimate a non fuzzy difference-in-difference (de Chaisemartin and d'Haultfoeuille, 2018). This framework allows us to formally analyze the placement of bednets and test if pre-trends are parallel.

We restrict analysis to survey clusters whose nearest neighbor in the earliest wave was below $75 \%$, hence only excluding the minority whose nearest neighbor was already above. We define our treatment variable as $T_{c}=\mathbb{1}\left\{B_{n\left(c, \max w_{j}\right)} \geqslant 0.75\right\}$, meaning that treated survey clusters are those ones which ended up with a closest neighbor displaying a bednet coverage above $75 \%$ in the last wave of surveys. As all countries received the first grants of the Roll 
Back Malaria campaign after 2003, with the exception of Mali who started in 2002, the year 2003 can be seen as a natural choice for the beginning of the treatment. Yet we can flexibly explore alternative choices in the starting date $\tau$. We just have to adapt our previous specifications to a straightforward double difference setting:

$$
y_{i c m j t}=\alpha T_{c} \cdot \mathbb{1}\{t \geqslant \tau\}+\beta_{1} X_{1 i}+\beta_{2} X_{2 m}+\gamma_{j t}+\delta_{c}+\zeta Z_{n(c, t)}+\varepsilon_{i m c j t}
$$

$\alpha$ is then the difference-in-difference estimate of the drop in mortality achieved when shifting to high levels of bednet coverage. As our data covers many cohorts of children, we can also estimate a break-in-trend specification, using a linear spline function of time with a kink-point at $\tau$ :

$y_{i c m j t}=\alpha_{1} T_{c} \cdot[\mathbb{1}\{t \geqslant \tau\} \cdot(t-\tau)]+\alpha_{0} T_{c} \cdot[\mathbb{1}\{t<\tau\} \cdot(t-\tau)+\tau]+\beta_{1} X_{1 i}+\beta_{2} X_{2 m}+\gamma_{j t}+\delta_{c}+\varepsilon_{i m c j t}$

$\alpha_{0}$ then provides a test for the existence of pre-treatment difference in time trend between treated and non-treated clusters. $\alpha_{1}$ indicates a post-treatment divergence in mortality.

\section{Results and Discussion}

\subsection{Main estimates}

Table 3 reports the correlation between infant mortality and bednet ownership rate in nearest neighbor, from the estimation of Equation 1 (cluster fixed effects), separately for urban (left panel) and rural (right) areas. We also break down our estimations according to malaria prevalence, using the estimate for the year 2000 by the Malaria Atlas (Bhatt et al., 2015); we

classify areas where prevalence was above $37.5 \%$ as highly malarious areas (roughly half of our sample). In each column, the top panel displays the coefficient of the continuous measure 
of the neighbor coverage rate $B_{n(c, t)}$, and the bottom panel a split of the coverage rate into four brackets of equal range: under 25\%, 25-50\%, 50-75\% and above 75\%. All estimations directly control for the vaccination coverage in five categories, and the prenatal exam coverage in three categories; they also control for the average electrification in the neighborhood as well as average education and asset ownership (we construct these quantities using the same methodology as for the bednet coverage).

When we use a continuous measure of ownership rate, we find a correlation of -0.019 significant at $90 \%$ in both urban and rural areas. Yet, this apparent similarity hides distinct features. Among urban areas, the correlation is slightly more pronounced in places where malaria prevalence is lower, in column (2) compared to column (3); yet the difference is not significantly different from zero. Among rural areas, the opposite is observed: in lowly malarious places the coefficient of bednet coverage is a pure zero, whereas in highly malarious places it reaches -0.040 with $95 \%$ significance (the difference between the two coefficients is also significantly different from zero).

In these highly malarious rural areas, the bottom panel reveals that the relationship between infant mortality and bednet ownership rate is not linear. Compared to the first bracket, mortality is the same in the second bracket, and although the coefficient associated to the third bracket $([50 \% ; 74 \%])$ is negative $(-0.009)$, it is not significantly different from zero and is much below the one associated to [75\%;100\%], that reaches -0.031 . All the effect of bednet distribution is concentrated above the $75 \%$ threshold. This is consistent with results from the entomology literature: the number of mosquito starts decreasing when a large share of people in a community use ITNs (Hougard, 2008).

In the case of urban areas, given that country-level and subnational level estimations already questioned the existence of a negative correlation, the small magnitude of coefficients and their odd pattern makes us suspect the action of a spurious confounding factor. Selective migration to cities or proactive urban health policies make two good candidates. 


\subsection{Education, migration and city size}

We look at the heterogeneity of the effect of bednets according to the education of the mother. Combined with the urban/rural distinction, this line of heterogeneity allows us to address the the migration bias that could plague the causal interpretation of the correlation between bednet coverage and infant mortality. Indeed, one drawback of the specification using retrospective birth histories is abstracting from migration. We observe a given mother in a given cluster of the last DHS wave and we implicitly assume that all her children are born in this same cluster, which is not true if the mother migrated in the course of her reproductive life. This generates measurement errors: we attribute a wrong bednet coverage to first-borns. Furthermore, if migrations are somehow related to both infant mortality and bednet distribution, this could potentially bias our results. Let us consider for instance rural-urban migration. If cities with better access to health care, including bednets, attract more rural migrants, we would observe a decrease in infant mortality correlated with bednet coverage due to those mothers who moved from rural to urban areas between their first- and later-born children. We expect rural areas to be less affected by this kind of migration bias.

Table 4 reports heterogenous estimates by maternal education for cluster fixed effects estimates, for areas with high malaria prevalence. ${ }^{13}$ The Online Appendix Table A.5 reports the same results for areas with low prevalence. We define three levels of education: none, primary and secondary; primary means having at least completed one year of formal education at primary level, while secondary means having at least completed one year at secondary level.

In highly malarious rural places, the relationship between bednet coverage and infant mortality is much stronger and only significant for non-educated mothers. For them, infant mortality is 4 percentage points lower when bednet coverage exceeds $75 \%$. In rural areas with low prevalence, the same pattern is found: the coefficient of bednet coverage is negative

\footnotetext{
${ }^{13}$ Estimates with mother fixed effects, available on request, are not different.
} 
only for non-educated mothers, yet remains not significantly different from zero. This result is quite reassuring with regards to migration bias, as we do not expect the non-educated mothers to be the most mobile women. They did not go to school, and their main potential move to join their husband after marriage should have preceded the birth of their first child.

In urban areas, the effect of bednets is conversely driven by mothers who attended secondary schools. In high prevalence places, the coefficient of bednet coverage is negative only for secondary-level mothers, even if none of the coefficients pass the bar of statistical significance (column 1c, Table 4); in low prevalence places, only one coefficient is significant at 90\% confidence, the one associated to a bednet coverage above $75 \%$, again for secondary-level (column 1c, Online Appendix Table A.5). This is rather odd since these urban, educated women could probably afford anti-malaria treatments before the campaigns. Many of these educated mothers could be deemed to be city natives, or to have reached the city when starting middle or high school, hence before they started having children. Yet they could also have moved from one city to another, and might have preferred moving to cities where the health environment was improving, correlatively with an increase in bednet coverage, either through the action of health facilities or through private purchases by middle class households. In such cities, educated women could also be more often doctors, nurses, or even teachers and social workers, who contributed to an accelerated diffusion of bednets and are also better able to take care of their own children. Urban areas selected in our sample are already larger than average, and within the sample large cities display lower malaria prevalence (Pond, 2013). ${ }^{14}$ In Online Appendix Table A.6, we checked for heterogeneity according to city size, making use of geo-referenced population census data. All the negative correlation between infant mortality and bednet coverage comes from the largest cities (above 150,000

\footnotetext{
${ }^{14}$ The urban environment is not too favorable to mosquitoes and/or low malaria suitability is more favorable to city growth. It is unclear whether the Malaria Atlas accurately estimates the prevalence in large cities, yet cities above 150,000 inhabitants display lower malaria prevalence by 13 pp compared to rural areas.
} 
inhabitants at the latest population census). ${ }^{15}$ This result is consistent with a story whereby large cities have more active health policies and attract health-sensitive educated mothers (including the health professionals and social workers who implement these policies).

We conclude that in urban areas the correlation of bednet coverage with infant mortality should not be interpreted as a causal effect. In the remainder, we focus on rural areas, and specifically on highly malarious ones.

\subsection{Robustness and placebo test}

Table 5 checks the robustness of the bednet coverage effect in rural areas with high malaria prevalence. Column (1) reports the estimates of column (6) in Table 3, with cluster fixed effects. Column (2) tests for the influence of our population reweighing: probability weights are no longer corrected for the country-survey specific sample rates, and only original probability weights are applied; in each country, the sum of weights is equal to the sample size instead of the total population of children. Column (3) introduces mother fixed effects, as in Equation 2, and column (4) adds cluster specific time trends. Without population reweighing, the point estimates is slightly decreased to -0.029 instead of -0.040 , yet statistical precision is improved so that the bednet coverage effect remains highly significant. When mother fixed effects and cluster time trends are introduced, the point estimates are higher, although not significantly different. Estimates (not shown) do not change either when controls for luminosity in the area are introduced (lit/unlit dummy and light density per square kilometers), although on a reduced sample (we could not get data for Ghana, Senegal and Liberia).

The endogenous placement of bednets is of course a major threat to causal inference, and the control variables introduced in our main specifications only partially take care of it. For instance, one can imagine that campaigns targeted some areas depending on the prevalence

\footnotetext{
${ }^{15}$ No heterogeneity is found for rural areas, with respect to the size of the closest city (the median being 35,000 inhabitants rather than 150,000).
} 
rate, the population density, the road accessibility or the economic vulnerability, all variables that could also impact the dynamics of infant mortality. The variation in bednet coverage in a region may still capture specific trends in infant mortality. We then conduct a placebo test on older birth cohorts, born before the Roll-Back Malaria initiative started. ${ }^{16}$ In the first DHS wave, we select the survey clusters which are the nearest neighbor of at least one last wave cluster of our analysis sample. We lead the date of birth of children by the time difference between last wave and first wave. Running our main specification on this placebo sample, we are able to test if the bednet coverage many years after a birth is related to the probability to die before age one. If there is a correlation, it cannot be driven by the causal impact of bednets on survival. It has to be the case that areas where more bednets have been distributed also have specific dynamics in mortality that are not captured by linear time trends. Table 6 shows the results of this placebo test for highly malarious rural areas, and with either cluster or mother fixed effects. In both cases, the coefficient on the two last brackets of bednets coverage are very close to zero. This test on older cohorts provides a first piece of evidence that anti-malaria campaigns have not been more intense in regions with better trends in infant mortality. Binary treatment models thereafter will also test for pre-trends, again on children born before the campaigns, but observed in the last wave of surveys.

\subsection{Other outcomes: fertility and neonatal mortality}

We also looked at the impact on fertility and neonatal mortality (Online Appendix Table A.7). For fertility, we find no significant impact of bednet coverage on birth rate. If malaria control impacts child survival, the effect is certainly too recent for fertility behaviors to have adjusted. For neonatal mortality, the protection of mothers during childbearing might matter

\footnotetext{
${ }^{16}$ We would also have liked to look at health outcomes that should not be impacted by bednets distribution. Yet, only fertility (see below) and mortality are available on a retrospective basis. Besides, alleviating the need for anti-malaria treatments could free resources to other uses, in a competing risks scenario.
} 
for the survival of the child just after birth. We could then find the same patterns as for infant mortality although with smaller magnitudes, because the specifications are in levels and the baseline neonatal mortality is lower. This is indeed what we find. The coefficients of bednet coverage are just about half of the coefficients found for infant mortality, and are significantly different from zero at $95 \%$ confidence. In our data, baseline neonatal mortality is estimated to account for one half of infant mortality, quite consistently with United Nations' estimates for Sub-Saharan Africa (UN Inter-agency Group for Child Mortality Estimation, 2015). Yet we do not put too much emphasis on this result, for the distinction between neonatal and infant mortality might not be very clear, given the uncertainties attached to the reporting of age at death, whose distribution displays some heaping at 0, 6, 9 and 12 months. Last, we could not identify a robust effect on under five mortality; to measure this outcome correctly, we must restrict the analysis sample to children born five years before the date of the survey, so that in most countries children born after 2007, when the international campaign accelerated, are selected out.

\subsection{Binary treatment}

We now turn to our binary treatment model, where treated survey clusters are those ones which ended up with a closest neighbor displaying a bednet coverage above $75 \%$ in the last wave of surveys. We start by describing the placement of bednets, comparing treated and non-treated clusters. Within countries, distribution targeted rural areas: about two thirds of rural clusters are treated vs. one half of urban clusters (yet remember that the analysis sample already excludes two thirds of rural areas vs. one third of urban, which do not have a close enough neighbor). Next, treated rural villages display higher infant mortality and deprivation (less access to health care, less education), and are further away from the biggest city (see balancing tests in Online Appendix Table A.8).

Table 7 reports the results of the break-in-trends specification of Equation 5, with 2003 as 
the kink-point year, and with and without mother fixed effects. The left-hand panel reports estimates for all rural areas, and the two right-hand panels break them down according to malaria prevalence.

The binary treatment models with cluster fixed effects provide a corroboration of our previous results with a continuous treatment. After 2003, treated rural villages saw infant mortality decrease at an accelerated pace of -0.43 percentage points per year, compared to non-treated. Once again, all the effect comes from highly malarious areas, where the pace of decrease reached -0.69 pp per year. No break in trend is identified in rural villages with low malaria prevalence. Another important result is that before 2003, there is no difference in trends between treated and non-treated clusters; the difference in pre-2003 trends is not significant and very close to zero. The null hypothesis of no change in the difference in trends can be rejected at 95\% confidence (see row "H0: Equal Trends"). The existence of a causal link in rural places is thus supported by the fact that trends start to differ only once large-scale ITN distribution campaigns have been launched. Figure 3 illustrates this result graphically, along with an event study analysis of the difference in infant mortality between treated and non-treated. Limited statistical precision makes that year-to-year differences are not significant, yet the evolution across time is regular enough and the break in trend is clear. The models with mother fixed effects confirm this set of results. For highly malarious rural areas, the post-2003 difference in trends reduces to -0.53 pp per year but is still significant at $90 \%$ confidence. The slight loss in precision makes that the test for the double-difference in trend is only passed at $88 \%$ confidence.

For the sake of space, we do not report the estimation of the double-difference models of Equation 4. Figure A.2 in Online Appendix depicts the estimates of the $\alpha$ coefficient for years $\tau$ going from 1999 to 2011, with cluster fixed effects. For rural areas, the double difference turns significantly negative for $\tau=2005$ and stabilizes around -2 percentage points for all $\tau \geq 2005$. For highly malarious rural areas, the same double difference reaches a maximum 
of $-3.7 \mathrm{pp}$ for $\tau=2007$ (-3 pp for 2006 and $-2.6 \mathrm{pp}$ for 2008). ${ }^{17}$

To take stock, the binary treatment models quite corroborate the causal interpretation of the effect of bednets on infant mortality in highly malarious rural areas. ${ }^{18}$

\subsection{Discussion: orders of magnitude}

We here briefly wrap up the evidence obtained and discuss orders of magnitude.

The coefficient that we identified for the linear association of infant mortality with the bednet coverage rate is close to -0.019 (Table 3). Because the time variation of bednet coverage is only observed in the neighborhood, and spatial correlation is imperfect, we may think that the former coefficient is a lower bound (underestimation should be at most $40 \%$ if $\rho>0.7)$. Anyway, even this lower bound suggests that the universal distribution of bednets (bednet coverage reaching 100\%) would result in a mortality decrease of $1.9 \mathrm{pp}$, equivalent to $17 \%$ of the initial mortality rate (around 11\%, for children born 1998 to 2003 in rural areas). We then showed that this estimate was mixing a zero effect in areas with low malaria prevalence, and a doubled effect (-0.040) in highly malarious villages. In the latter, with universal distribution infant mortality would then drop by 4 pp, or $35 \%$ of baseline mortality (estimated around 11.5\%). Our estimates also suggest that only villages for which the neighbors' bednet coverage went above $75 \%$ benefited from a significant drop in infant mortality, of around 3.1 pp. Double difference estimates also suggest that children born after 2006 in treated villages enjoyed a drop of 3 pp. If we assimilate this effect to quasi-universal distribution, we still get an impact that reduces mortality by more than $25 \%$ compared to baseline. If we retain mother fixed effect estimates, we get even higher impacts: -2.5

\footnotetext{
${ }^{17}$ Dolan et al. (2019) use DHS data from the Democratic Republic of Congo and implement a doubledifference strategy based on the date of the bednets distribution campaigns in each province, from September 2009 to November 2012. Like us, they find no effect in areas with low malaria ecology, and estimate a 3.7 pp reduction in under-five mortality, equivalent to $41 \%$ of the baseline.

${ }^{18}$ If needed, the same models estimated for urban areas (not shown) also question the robustness of the effect in this context.
} 
pp (23\%) for rural areas as a whole (coefficient not shown in Tables), and -5.5 pp (50\%) in highly malarious villages. If we refer to the Cochrane review of RCTs that we already mentioned, the village-level universal distribution of ITNs is credited with a reduction of child mortality by 14 to $29 \%$ compared to no nets (Lengeler, 2004; Richardson et al., 2018). Our estimates then clearly lie at the upper bound of this interval and even beyond. The worry that optimistic RCTs results cannot be reached at scale seems to be misplaced. In fact, RCTs are more conservative than expected: as already underlined above, they were implemented in quite favorable contexts, where baseline mortality was low and/or malaria was not endemic. Our results suggest that the impact of ITNs could be higher, especially in highly malarious places.

Can we calculate the contribution of the bednets campaigns to the overall mortality decrease? Our own results were also obtained on a selected sample of better off localities: closer to the biggest city in their country, and with more access to education and health services. When we focus on highly malarious areas, they also exclude all children from the Democratic Republic of Congo, because of a too small sample size, and two countries where malaria prevalence is low, Rwanda and Senegal (see Online Appendix Table A.4). Extrapolation is quite risky in this context. Yet it is not obvious that the effect of bednet coverage is lower in the non-selected areas. Online Appendix Table A.9 presents the results of a simple extrapolation exercise, based on reweighing by a propensity score. In a first stage, we estimate a probit model for selection into the sample, with distance to the biggest city, mother's and husband's education, access to electricity and assets' ownership as explanatory variables, as well as country fixed effects (we exclude vaccination and prenatal care to avoid endogeneity concerns, yet this makes little difference in practice). In a second stage, we run again the models of Table 3 on rural areas, with probability weights divided by the first stage estimated probability. This amounts to assuming that all the heterogeneity in the effect of bednet coverage is explained by the set of observables included in the propensity score, and 
nothing else. In this case, the results are very clear: the correlation between infant mortality and bednet coverage doubles, both for rural areas as a whole and for the highly malarious subsample. Coefficients raise respectively from -0.0191 to -0.0390 and from -0.0402 to -0.0914 . This means that the selected children that are observationally close to the non-selected, i.e. from under-represented countries and/or in isolated villages, have even more benefited from the massive distribution of bednets. We still acknowledge that remote villages in countries

like DRC could very well have other unobserved characteristics that mitigate the benefits of bednets. We then refrain from using these coefficients for extrapolating the bednets-induced mortality decrease. More conservatively, let us retain the effects estimated from our selected sample and consider that they apply to the rest of rural areas. While the bednet coverage rate varied by some 45 percentage points, the contribution of bednets would then lie between $18 \%$ and $33 \%$ of the overall reduction in infant mortality (of about $5 \mathrm{pp}$ ), which is already not bad.

\section{Conclusion}

Using an original measure of the variation in bednet coverage, this paper shows that antimalaria campaigns in Africa over the past 15 year are strongly correlated to a reduction in infant mortality. The magnitude of the correlation turns out to lie above the experimental estimates of the protective efficacy of bednets. This conclusion was not obvious ex-ante. On the one hand, medical RCTs could lack external validity, either because they were implemented under highly controlled conditions (upward bias), or because they took place in specific settings with relatively low mortality and low malaria prevalence (downward bias). On the other hand, bednets distributions during the period 2000-2015 could have favored areas with better pre-existing trends in mortality, and could have been coupled with other health interventions. To isolate a causal effect of bednets, we extensively control for con- 
founders and carefully analyze trends.

We do not identify any causal impact on urban areas, where the pattern of correlations that we found can be interpreted as the combined result of active health policies and migration bias. In rural areas, we find no impact either on villages where malaria prevalence is low (below 37.5\%). We cannot exclude that our methodology is too coarse to detect small impacts in less needy contexts.

In contrast, we find that causal inference is very credible for the survival of children living in highly malarious areas where bednet coverage reached a high level in the neighborhood, above $75 \%$. This effect is very robust, and is concentrated on mothers with no education. Its magnitude is also quite large: universal distribution of insecticide-treated-nets (ITNs) would result in a 3 to 5 percentage points reduction of mortality in highly malarious villages, i.e. 25 to $50 \%$ of initial mortality, while most RCTs rather range between 15 and $30 \%$.

This paper highlights the trade-off between internal and external validity when assessing the impact of malaria control efforts on infant mortality.

\section{References}

Apouey, B.H., G. Picone, J. Wilde, J. Coleman, and R.M. Kibler, "Paludisme et anémie des enfants en Afrique subsaharienne : effet de la distribution de moustiquaires," Revue Economique, 2017, 68(2), 163-197.

Bhatt, S., D. J. Weiss, E. Cameron, D. Bisanzio, B. Mappin, U. Dalrymple, K. E. Battle, C. L. Moyes, A. Henry, P. A. Eckhoff, E. A. Wenger, O. Briet, M. A. Penny, T. A. Smith, A. Bennett, J. Yukich, T. P. Eisele, J. T. Griffin, C. A. Fergus, M. Lynch, F. Lindgren, C. L. J. Murray J. M. Cohen and, D. L. Smith, S. I.Hay, R. E. Cibulskis, and P.W. Gething, "The effect of malaria control on Plasmodium falciparum in Africa between 2000 and 2015," Nature, 2015, 526, 207-211.

Black, R., S. Cousens, H. Johnson, J. Lawn, I. Rudan, D. Bassani, P. Jha, H. Campbell, C. Fischer Walker, R. Cibulskis, T. Eisele, L. Liu, and C. Mathers, "Global, regional, and national causes of child mortality in 2008: a systematic analysis," The Lancet, 2014, 375 No 9730, 1969-1987.

Bleakley, H., "Malaria Eradication in the Americas: A Retrospective Analysis of Childhood Exposure," American Economic Journal: Applied Economics, 2010, 2, 1-45. 
Cohen, J. and P. Dupas, "Free Distribution of Cost-Sharing? Evidence from a Randomized Malaria Prevention Experiment," Quarterly Journal of Economics, 2010, 125 (1), $1-45$.

Cutler, D., W. Fung, M. Kremer, M. Singhal, and T. Vogl, "Early-life Malaria Exposure and Adult Outcomes: Evidence from Malaria Eradication in India," American Economic Journal: Applied Economics, 2010, 2, 72-94.

de Chaisemartin, C. and X. d'Haultfoeuille, "Fuzzy Differences-in-Differences," Review of Economic Studies, 2018, 85(2), 999-1028.

Dolan, C.B., A. BenYishay, K.A. Grépin, J.C. Tanner, A.D. Kimmel, and et al. D.C. Wheeler, "The impact of an insecticide treated bednet campaign on all-cause child mortality: A geospatial impact evaluation from the Democratic Republic of Congo," PLoS ONE, 2019, 14(2).

Dupas, P., "What Matters (and What Does Not) in Households' Decision to Invest in Malaria Prevention?," American Economic Review, 2009, 99 (2), 224-230.

_ , "Short-Run Subsidies and Long-Run Adoption of New Health Products: Evidence From a Field Experiment," Econometrica, 2014, 82 (1), 197-228.

Eisele, T.P., D.A. Larsen, P.A. Anglewicz, J. Keating, J. Yukich, A. Bennett, P. Hutchinson, and R.W. Steketee, "Malaria prevention in pregnancy, birthweight, and neonatal mortality: a meta-analysis of 32 national cross-sectional datasets in Africa," Lancet Infectious Diseases, 2012, 12, 942-949.

Goldsmith-Pinkham, P., I. Sorkin, and H. Swift, "Bartik Instruments: What, When, Why, and How," Working Paper 24408, National Bureau of Economic Research 2018.

Hightower, A., R. Kiptui, A. Manya, A. Wolkon, J. L. Vanden Eng, M. Hamel, A. Noor, S. K. Sharif, R. Buluma, J. Vulule, K. Laserson, L. Slutsker, and W. Akhwale, "Bed net ownership in Kenya: the impact of million free bed nets," Malaria Journal, 2010, 9 (183).

Hoffmann, V., "Intrahousehold Allocation of Free and Purchases Mosquito Nets," American Economic Review: Papers 83 Proceedings, 2010, 99 (2), 236-241.

Hougard, J-M, "Les moustiquaires impregnees," Pour la science, 2008, 366, 48-52.

Kesteman, T., M. Randrianarivelojosia, and C. Rogier, "The protective effectiveness of control interventions for malaria prevention: a systematic review of the literature," F1000Research, 2017, 6:1932.

Kudamatsu, M., T. Persson, and D. Stromberg, "Weather and Infant Mortality in Africa," Working Paper, 2012. 
Kuecken, M., J. Thuilliez, and M.A. Valfort, "Disease and Human Capital Accumulation: Evidence from the Roll Back Malaria Partnership in Africa," Working Paper, 2017.

Lengeler, C., "Insecticide-treated bed nets and curtains for preventing malaria," Cochrane Database of Systematic Reviews, 2004.

Lim, S.S., N. Fullman, A. Stokes, N. Ravishankar, F. Masiye, and C.J.L. Murray amd E. Gakidou, "Net Benefits: A Multicountry Analysis of Observational Data Examining Associations between Insecticide-Treated Mosquito Nets and Health Outcomes," PLoS Medicine, 2011, 8(9).

Lucas, A., "Malaria Eradication and Educational Attainment: Evidence from Paraguay and Sri Lanka," American Economic Journal: Applied Economics, 2010, 2, 46-71.

Pond, B.S., "Malaria indicator surveys demonstrate a markedly lower prevalence of malaria in large cities of sub-Saharan Africa," Malaria Journal, 2013, 12 (313).

Richardson, M., C. Lengeler, and J. Pryce, "Insecticide-treated nets for preventing malaria," Cochrane Database of Systematic Reviews, 2018.

Tarozzi, A., A. Mahajant, B. Blackburn, D. Kopf, L. Krishnan, and J. Yoong, "Micro-loans, Insecticide-Treated Bednets, and Malaria: Evidence from a Randomized Controlled Trial in Orissa, India," American Economic Review, 2014, 104 (7), 1909-1941.

UN Inter-agency Group for Child Mortality Estimation, "Levels and Trends in Child Mortality," United Nations Children's Fund, 2015.

UNICEF, "Generation 2030," UNICEF publication, Division of Data, Research and Policy, 2014.

United Nations, "The Millenium Development Goals Reports," United Nations publication, ESA, 2015.

Venkataramani, A., "Early life exposure to malaria and cognition in adulthood: Evidence from Mexico," Journal of Health Economics, 2010, 31(5), 691-780.

WHO, "World Malaria Report," WHO publication, 2015.

Wilde, J., B.H. Apouey, J. Coleman, and G. Picone, "The Effect of Antimalarial Campaigns on Child Mortality and Fertility in Sub-Saharan Africa," Working Paper, 2017.

Yazoume, Y., T.P. Eisele, E. Eckert, E. Korenromp, J.A. Shah, C.L. Hershey, E. Ivanovich, H. Newby, L. Carvajal-Velez, M. Lynch, R. Komatsu, R.E. Cibulskis, Z. Moore, and A. Bhattarai, "Framework for Evaluating the Health Impact of the Scale-Up of Malaria Control Interventions on All-Cause Child Mortality in Sub-Saharan Africa," American Journal of Tropical Medicine and Hygiene, 2017, 97(Suppl 3), 9-19. 
Figures and Tables 
Figure 1: Panel analysis: country-level

All areas

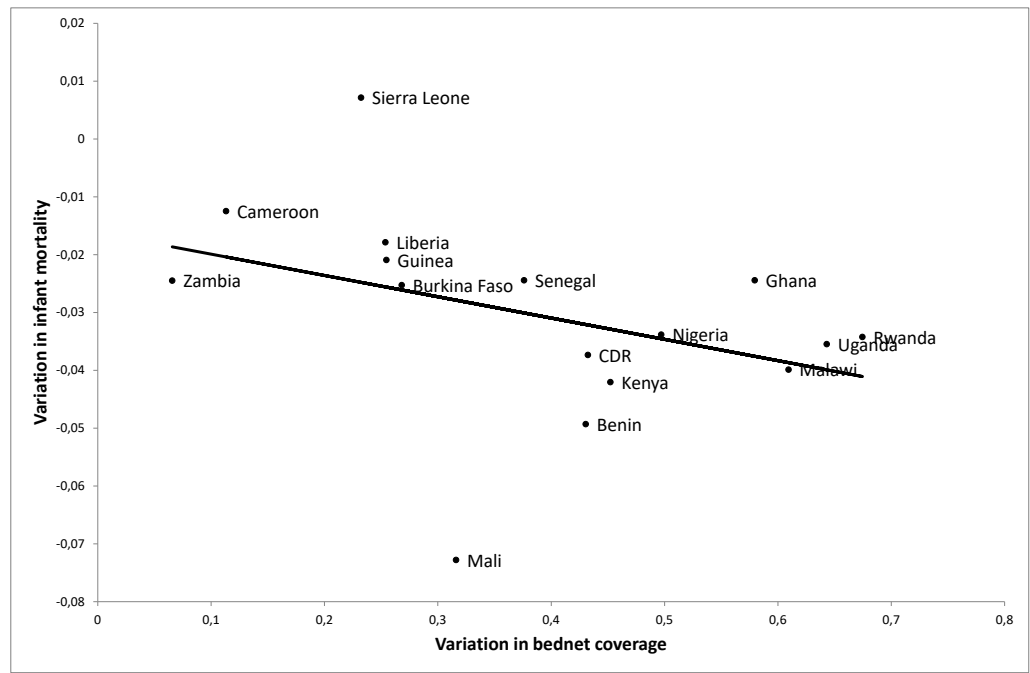

\section{Urban areas}

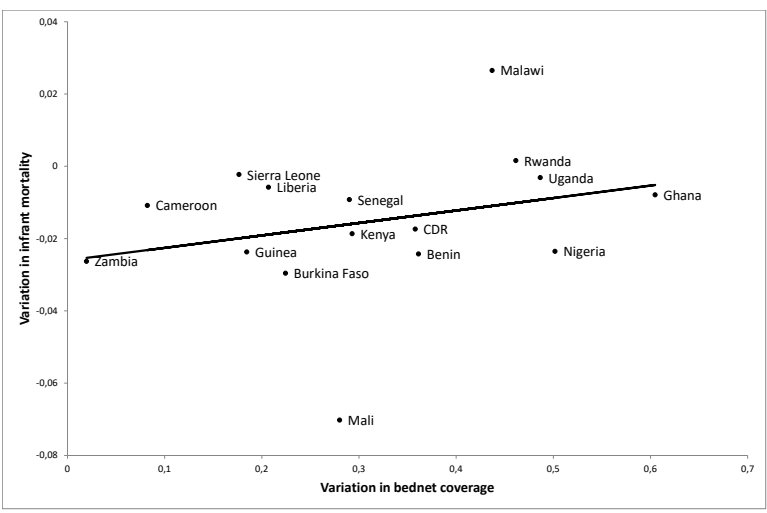

Rural areas

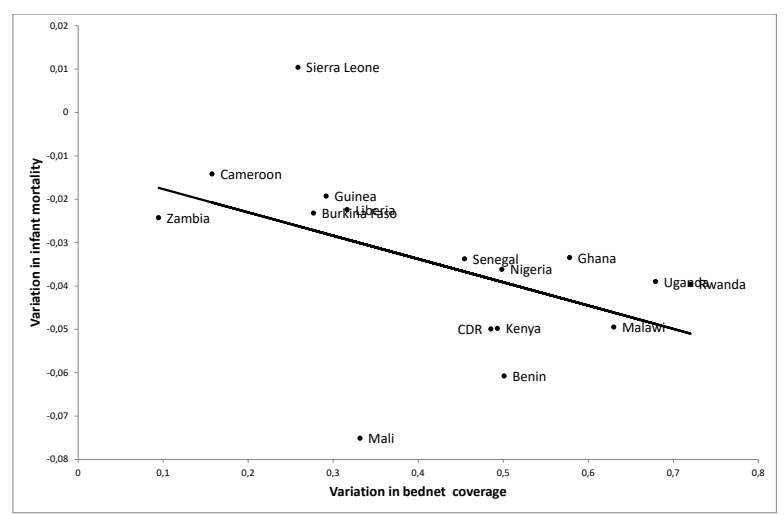

Notes: The figures plot the variation in bednet coverage (share of mothers 15-49 y.o. having at least one bednet at home) on the $x$ axis and the variation in infant mortality on the $y$ axis, between the first (2000-2008) and last (2010-2014) waves of DHS. Infant mortality rates are computed on children born 1-4 years before survey date. Each dot corresponds to a country. The slopes correspond to a linear regression fit. In the top figure, the slope is -0.036 (s.e. 0.023). In the bottom figures, the slopes are respectively +0.034 (s.e. 0.032) for urban areas, and -0.054 (s.e. 0.026) for rural areas. 
Figure 2: Bednets spatial distribution [requires color]
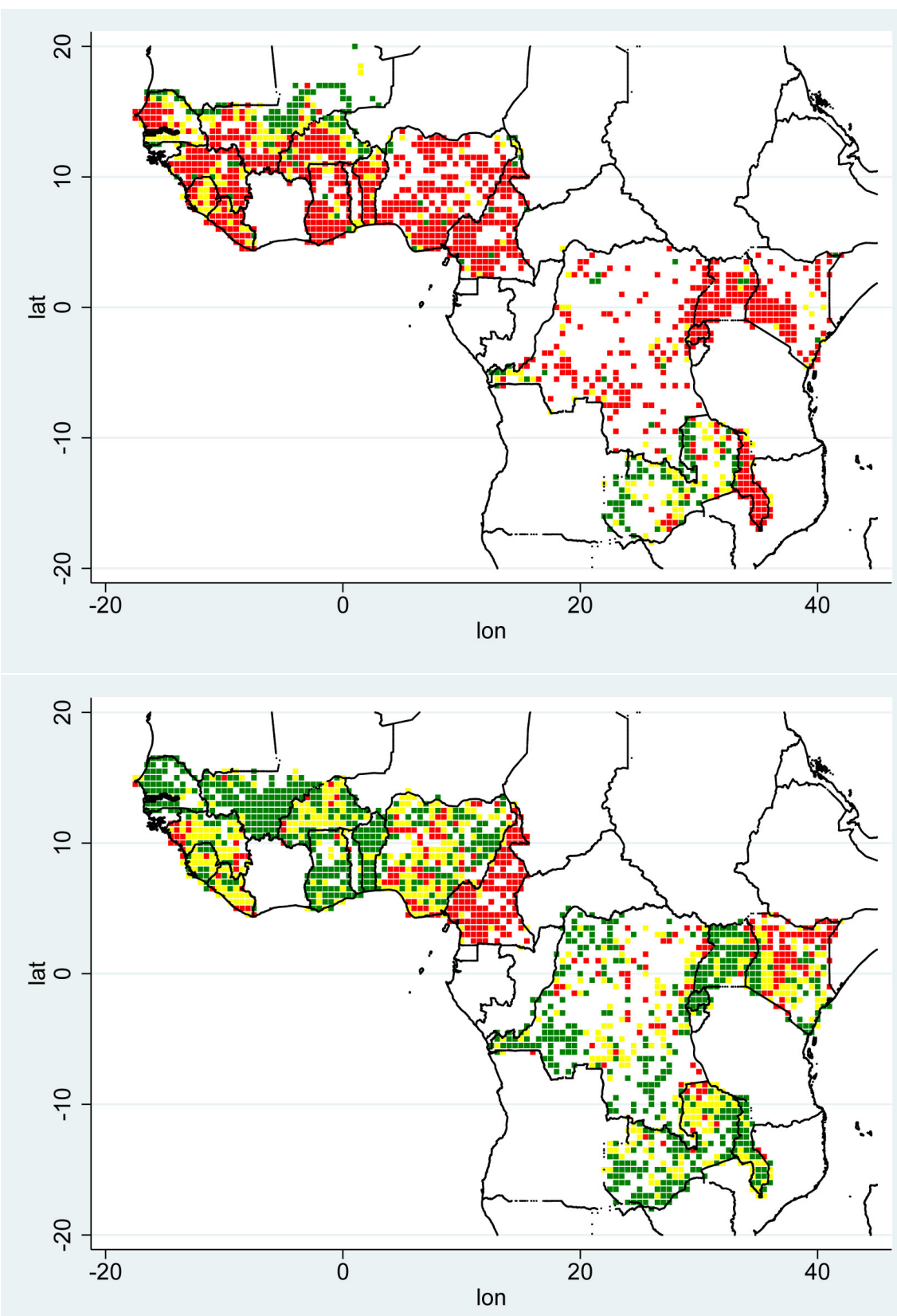

Notes: The two maps display the share of 15-49 y.o. mothers having at least one bednet at home, respectively in the first (2000-2008) and in the second (2010-2014) DHS waves of each country, in $0.5 \times 0.5$ degree squares. Red dots have less than $50 \%$ coverage, yellow 50 to $74 \%$, and green $75 \%$ or more. 


\section{Figure 3: Event Study Analysis with Binary Treatment in Rural Areas}

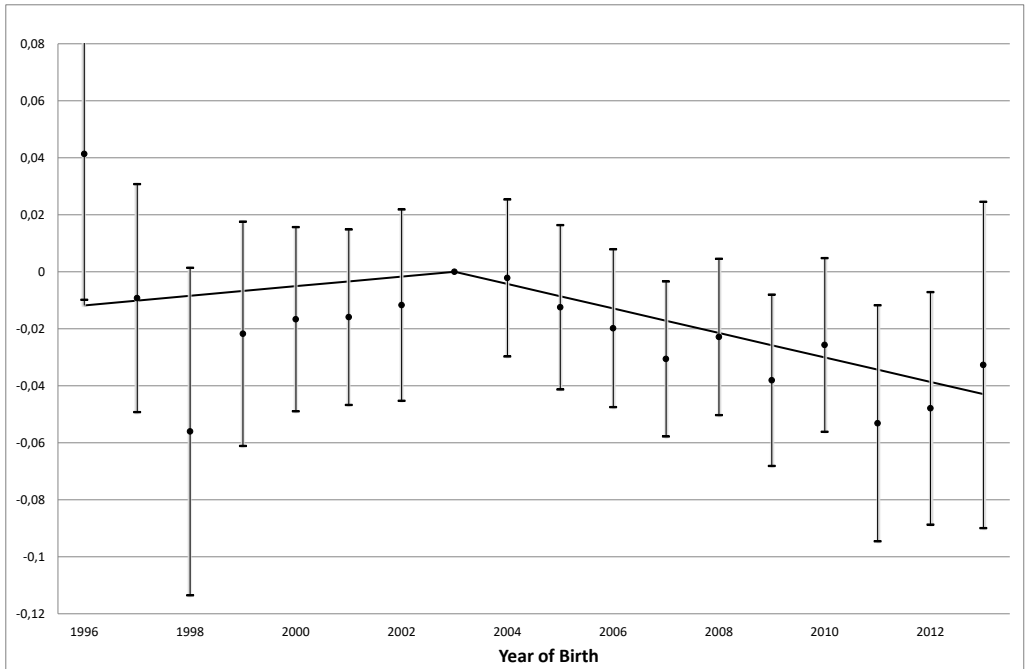

Notes: The graph displays the coefficients of the treatment dummy interacted with the year of birth of the child, with confidence intervals at $95 \%$. The plain line show the difference in trend between treated and non-treated, from a linear spline function with a kink point in birth year 2003: coefficients displayed in Table 7 column (1) (cluster FE estimates). 
Table 1: Correlation between bednet coverage and infant mortality at subnational level

\begin{tabular}{lccc}
\hline \hline Dep. var.: & \multicolumn{3}{c}{ Variation in infant mortality } \\
Indep. var.: & \multicolumn{2}{c}{ Variation in bednet coverage } \\
Sample: & All & Urban & Rural \\
& $(1)$ & $(2)$ & $(3)$ \\
\hline $0.5 \times 0.5$ degree & -0.0125 & +0.0410 & -0.0162 \\
& $(0.0135)$ & $(0.0360)$ & $(0.0112)$ \\
N (average) & 1,168 & 462 & 1,027 \\
N squares (average) & 1,736 & 758 & 1,632 \\
\hline $0.25 \times 0.25$ degree & -0.0155 & +0.0332 & -0.0232 \\
& $(0.0107)$ & $(0.0316)$ & $(0.0112)$ \\
N (average) & 1,738 & 542 & 1,369 \\
N squares (average) & 3,445 & 1,095 & 3,073 \\
\hline $0.1 \times 0.1$ degree & -0.0180 & +0.0263 & -0.0327 \\
& $(0.0148)$ & $(0.0370)$ & $(0.0221)$ \\
N (average) & 1,722 & 584 & 1,065 \\
N squares (average) & 5,941 & 1,528 & 4,850 \\
\hline Country FE & Yes & Yes & Yes \\
\hline
\end{tabular}

Sample: Within-country $d \times d$ degree squares averages.

Sample for mortality rates: Children born 1-4 years before survey date.

Notes: Bednet coverage if the share of mothers 15-49 y.o. having at least one bednet at home. Reported coefficients come from

a linear regression of the variation in infant mortality in one square on the variation of bednet coverage in the same square, with country FE included. We report the average of 25 coefficient estimates from 25 different grids (shifted by $k \times 0.2 \times d$ in latitude and $l \times 0.2 \times d$ in longitude, with $0 \leq k \leq 4$ and $0 \leq l \leq 4)$. N squares = total number of squares in the grid with at least one observation from the last survey wave; $\mathrm{N}=$ number of squares with non-missing variations. Probability weights adjusted for country population are used. Average standard errors of coefficients are between parenthesis (clustered at country-level). 
Table 2: Spatial correlation of bednet coverage expansion

\begin{tabular}{lccc}
\hline \hline Dep. var.: & \multicolumn{3}{c}{ Variation in bednet coverage } \\
Indep. var.: & \multicolumn{2}{c}{ Average var. in neighboring squares } \\
Sample: & All & Urban & Rural \\
& $(1)$ & $(2)$ & $(3)$ \\
\hline $0.5 \times 0.5$ degree & +0.592 & +0.249 & +0.577 \\
& {$[0.445 ; 0.739]$} & {$[0.110 ; 0.388]$} & {$[0.422 ; 0.733]$} \\
$\mathrm{N}$ (average) & 1,150 & 398 & 1,007 \\
$\mathrm{~N}$ squares (average) & 1,736 & 758 & 1,632 \\
\hline $0.25 \times 0.25$ degree & +0.628 & +0.392 & +0.698 \\
& {$[0.470 ; 0.786]$} & {$[0.0147 ; 0.636]$} & {$[0.555 ; 0.842]$} \\
$\mathrm{N}$ (average) & 1,645 & 353 & 1,284 \\
$\mathrm{~N}$ squares (average) & 3,445 & 1,095 & 3,073 \\
\hline $0.1 \times 0.1$ degree & +0.405 & +0.181 & +0.376 \\
& {$[0.213 ; 0.597]$} & {$[-0.261 ; 0.622]$} & {$[0.178 ; 0.574]$} \\
$\mathrm{N}$ (average) & 863 & 174 & 501 \\
$\mathrm{~N}$ squares (average) & 5,941 & 1,528 & 4,850 \\
\hline Country FE & Yes & Yes & Yes
\end{tabular}

Sample: Within-country $d \times d$ degree squares averages.

Notes: Bednet coverage if the share of mothers 15-49 y.o. having at least one bednet at home. Reported coefficients come from a linear regression of the variation in bednet coverage in one square on the average variation of bednet coverage in the eight neighboring squares, with country FE included. We report the average of 25 coefficient estimates from 25 different grids (shifted by $k \times 0.2 \times d$ in latitude and $l \times 0.2 \times d$ in longitude, with $0 \leq k \leq 4$ and $0 \leq l \leq 4)$. N squares $=$ total number of squares in the grid with at least one observation from the last survey wave; $\mathrm{N}=$ number of squares with non-missing variations. Probability weights adjusted for country population are used. Standard errors are clustered at country-level. Confidence interval at $95 \%$ in brackets. 
Table 4: Heterogeneity according to mother education in highly malarious areas

\begin{tabular}{|c|c|c|c|c|c|c|}
\hline \multirow{4}{*}{$\begin{array}{l}\text { Dep. var. : } \\
\text { Indep. var.: } \\
\text { Sample: }\end{array}$} & \multicolumn{6}{|c|}{ Died before age 1} \\
\hline & \multicolumn{6}{|c|}{ Bednet coverage in nearest neighbor } \\
\hline & \multicolumn{3}{|c|}{ Urban } & \multicolumn{3}{|c|}{ Rural } \\
\hline & $\begin{array}{l}\text { None } \\
(1 \mathrm{a})\end{array}$ & $\begin{array}{l}\text { Primary } \\
\quad(1 \mathrm{~b})\end{array}$ & $\begin{array}{l}\text { Secondary } \\
\quad(1 c)\end{array}$ & $\begin{array}{l}\text { None } \\
(2 \mathrm{a})\end{array}$ & $\begin{array}{l}\text { Primary } \\
(2 \mathrm{~b})\end{array}$ & $\begin{array}{l}\text { Secondary } \\
\quad(2 \mathrm{c})\end{array}$ \\
\hline \multirow[t]{2}{*}{ Continuous } & +0.0162 & +0.0218 & -0.0173 & $-0.0515^{* * *}$ & -0.0296 & +0.0085 \\
\hline & $(0.0143)$ & $(0.0161)$ & $(0.0154)$ & $(0.0182)$ & $(0.0195)$ & $(0.0295)$ \\
\hline \multirow[t]{2}{*}{$25-49 \%$} & -0.0083 & +0.0007 & $-0.0178^{*}$ & +0.0063 & -0.0071 & +0.0030 \\
\hline & $(0.0143)$ & $(0.0153)$ & $(0.0097)$ & $(0.0099)$ & $(0.0136)$ & $(0.0141)$ \\
\hline \multirow[t]{2}{*}{$50-74 \%$} & -0.0082 & -0.0061 & -0.0150 & -0.0126 & -0.0022 & +0.0192 \\
\hline & $(0.0147)$ & $(0.0140)$ & $(0.0098)$ & $(0.0108)$ & $(0.0134)$ & $(0.0216)$ \\
\hline \multirow[t]{2}{*}{$75-100 \%$} & -0.0081 & +0.0280 & -0.0197 & $-0.0398 * * *$ & -0.0228 & +0.0118 \\
\hline & $(0.0135)$ & $(0.0225)$ & $(0.0132)$ & $(0.0151)$ & $(0.0150)$ & $(0.0232)$ \\
\hline Children controls & \multicolumn{6}{|c|}{ Mother age at birth, Child sex } \\
\hline Neighbor controls & \multicolumn{6}{|c|}{ Average of household controls, Vaccination and Prenatal care } \\
\hline Birthyear-country FE & Yes & Yes & Yes & Yes & Yes & Yes \\
\hline Cluster FE & Yes & Yes & Yes & Yes & Yes & Yes \\
\hline N children & 13,849 & 9,968 & 14,208 & 22,568 & 18,973 & 4,638 \\
\hline $\mathrm{N}$ mothers & 5,502 & 4,255 & 7,484 & 7,849 & 6,531 & 2,104 \\
\hline $\mathrm{N}$ clusters & 1,038 & 1,038 & 1,038 & 859 & 859 & 859 \\
\hline \multicolumn{7}{|c|}{$\begin{array}{l}\text { Sample: Areas with malaria prevalence above } 37.5 \% \text { in } 2000 \text {, according to the Malaria Atlas (Bhatt et al., 2015). Survey clusters } \\
\text { ff DHS last wave whose two nearest neighbors in first and last wave and of the same urban/rural status are no further than } 10 \\
\text { sm. Children of mothers } 15-49 \text { y.o., born not earlier than } 5 \text { years before the first survey date. }\end{array}$} \\
\hline \multicolumn{7}{|c|}{$\begin{array}{l}\text { Notes: Mother education: highest level attained. Children controls, Vaccination and Prenatal care, and Birthyear-country FE } \\
\text { are interacted with mother education dummies. Standard errors in parentheses clustered at survey cluster (PSU) level. *** }\end{array}$} \\
\hline
\end{tabular}


Table 5: Robustness of the bednet coverage effect in highly malarious rural areas

\begin{tabular}{lcccc}
\hline \hline Dep. var. : & \multicolumn{5}{c}{ Died before age 1 } \\
Indep. var.: & \multicolumn{4}{c}{ Bednet coverage in nearest neighbor } \\
& $(1)$ & $(2)$ & $(3)$ & $(4)$ \\
\hline Continuous & $-0.0402^{* * *}$ & $-0.0292^{* * *}$ & $-0.0557^{* * *}$ & $-0.0695^{* * *}$ \\
& $(0.0161)$ & $(0.0113)$ & $(0.0175)$ & $(0.0263)$ \\
\hline $25-49 \%$ & -0.0007 & -0.0050 & -0.0027 & -0.0094 \\
& $(0.0086)$ & $(0.0073)$ & $(0.0085)$ & $(0.0119)$ \\
$50-74 \%$ & -0.0090 & -0.0086 & -0.0153 & -0.0130 \\
& $(0.0095)$ & $(0.0075)$ & $(0.0106)$ & $(0.0144)$ \\
$75-100 \%$ & $-0.0313^{* *}$ & $-0.0238^{* * *}$ & $-0.0413^{* * *}$ & $-0.0515^{* * *}$ \\
& $(0.0123)$ & $(0.0089)$ & $(0.0132)$ & $(0.0205)$ \\
\hline Children controls & \multicolumn{4}{c}{ Mother age at birth, Child sex } \\
Household controls & Wealth, Electricity, Radio, Education \\
Neighbor controls & \multicolumn{4}{c}{ Av. of hh controls, Health policies } \\
Population weights & Yes & No & Yes & Yes \\
Birthyear-country FE & Yes & Yes & Yes & Yes \\
Cluster FE & Yes & Yes & Yes & Yes \\
Mother FE & No & No & Yes & Yes \\
Cluster linear time trend & No & No & No & Yes \\
N children & 46,196 & 46,196 & 42,517 & 42,517 \\
N mothers & 16,485 & 16,485 & 12,806 & 12,806 \\
N clusters & 859 & 859 & 859 & 859 \\
\hline
\end{tabular}

Sample: Areas with malaria prevalence above 37.5\% in 2000, according to the Malaria Atlas (Bhatt et al., 2015). Rural survey clusters of DHS last wave whose two rural nearest neighbors in first and last wave are no further than $10 \mathrm{~km}$. Children of mothers 15-49 y.o., born not earlier than 5 years before the first survey date.

Notes: Children controls: Set of dummies for mother age at birth and for child sex. Household controls: wealth score (7 assets), dummies for electricity and radio at home, dummies for mother completed primary and for completed secondary, and husband education in 4 levels. Health policies: vaccination ( 5 categories) and prenatal exam (3 categories) coverage in nearest neighbor. Probability weights adjusted for country population. Standard errors in parentheses clustered at survey cluster (PSU) level. *** $p<0.01, * * p<0.05, * p<0.10$. 
Table 6: Placebo in highly malarious rural areas

\begin{tabular}{lcc}
\hline $\begin{array}{l}\text { Dep. var.: } \\
\text { Indep. var.: }\end{array}$ & \multicolumn{2}{c}{$\begin{array}{c}\text { Died before age 1 } \\
(1)\end{array}$} \\
& +0.0054 & $(2)$ \\
\hline Continuous & $(0.0316)$ & +0.0070 \\
& -0.0053 & $(0.0310)$ \\
\hline $25-49 \%$ & $(0.0247)$ & -0.0039 \\
& +0.0144 & $(0.0251)$ \\
$50-74 \%$ & $(0.0257)$ & +0.0128 \\
& -0.0002 & $(0.0295)$ \\
$75-100 \%$ & $(0.0246)$ & +0.0023 \\
& \multicolumn{2}{c}{ Mother age at birth, Child sex } \\
\hline Children controls & Wealth, Electricity, Radio, Education \\
Household controls & Av. of hh controls, Health policies \\
Neighbor controls & Yes & Yes \\
Birthyear-country FE & Yes & Yes \\
Cluster FE & No & Yes \\
Mother FE & 11,361 & 10,325 \\
N children & 4,073 & 3,038 \\
N mothers & 253 & 253 \\
N clusters & & \\
\hline
\end{tabular}

Sample: Areas with malaria prevalence above 37.5\% in 2000, according to the Malaria Atlas (Bhatt et al., 2015). Rural survey clusters of DHS first wave whose nearest neighbor in first wave is no further than $10 \mathrm{~km}$, and which are the nearest neighbor $(<10 \mathrm{~km})$ of at least one last wave cluster of the analysis sample of Table 3. Children of mothers 15-49 y.o, born not earlier than 5 years before survey date minus the time difference between last wave and first wave (lead).

Notes: Placebo treatment variable is bednet coverage in nearest neighbor $(<10 \mathrm{~km})$ of the survey wave whose date is closest to the child date of birth plus the time difference between last wave and first wave (lead). The same lead applies to neighbor controls. Probability weights adjusted for country population. Standard errors in parentheses clustered at survey cluster (PSU) level. $* * * p<0.01,{ }^{* *} p<0.05, * p<0.10$. 
Table 7: Binary treatment models : 75\% coverage threshold

\begin{tabular}{|c|c|c|c|c|c|c|}
\hline \multirow{3}{*}{$\begin{array}{l}\text { Dep. var.: } \\
\text { Sample: }\end{array}$} & \multicolumn{6}{|c|}{ Died before age 1} \\
\hline & \multicolumn{2}{|c|}{ Rural All } & \multicolumn{2}{|c|}{ Rural Low Malaria } & \multicolumn{2}{|c|}{ Rural High Malaria } \\
\hline & (1) & (2) & (3) & (4) & (5) & (6) \\
\hline \multirow[t]{2}{*}{ T pre-2003 $\times$ Treated } & +0.0017 & -0.0002 & -0.0009 & -0.0024 & +0.0047 & +0.0024 \\
\hline & $(0.0022)$ & $(0.0023)$ & $(0.0024)$ & $(0.0027)$ & $(0.0033)$ & $(0.0033)$ \\
\hline \multirow[t]{2}{*}{ T post-2003 $\times$ Treated } & $-0.0043^{* * *}$ & $-0.0028^{*}$ & -0.0005 & +0.0016 & $-0.0069^{* * *}$ & $-0.0053^{* *}$ \\
\hline & $(0.0015)$ & $(0.0016)$ & $(0.0017)$ & $(0.0020)$ & $(0.0024)$ & $(0.0025)$ \\
\hline H0: Equal Trends (p-val.) & 0.049 & 0.423 & 0.812 & 0.324 & 0.018 & 0.118 \\
\hline Children controls & \multicolumn{6}{|c|}{ Mother age at birth, Child sex } \\
\hline Household controls & \multicolumn{6}{|c|}{ Wealth, Electricity, Radio, Education } \\
\hline Birthyear-country FE & Yes & Yes & Yes & Yes & Yes & Yes \\
\hline Cluster FE & Yes & Yes & Yes & Yes & Yes & Yes \\
\hline Mother FE & No & Yes & No & Yes & No & Yes \\
\hline $\mathrm{N}$ children & 83,151 & 75,700 & 39,391 & 35,449 & 43,756 & 40,247 \\
\hline $\mathrm{N}$ mothers & 31,044 & 23,593 & 15,423 & 11,481 & 15,620 & 12,211 \\
\hline $\mathrm{N}$ clusters & 1,779 & 1,779 & 969 & 969 & 810 & 810 \\
\hline
\end{tabular}

Sample: Rural survey clusters of DHS last wave whose nearest neighbor in first wave is (i) no further than $10 \mathrm{~km}$, and (ii) had bednet coverage lower than 75\%. Children of mothers 15-49 y.o., born not earlier than 5 years before the first survey date. The sample of clusters is a bit smaller than in previous tables because we restrict to clusters whose first wave neighbor was below $75 \%$.

Notes: The binary treatment is a dummy variable for the nearest neighbor $(<10 \mathrm{~km})$ in the last survey wave having reached a bednet coverage above 75\%. 2003 is the earliest date of the first Global Fund transfers to the countries (except Mali who received its first grant in 2002). Coefficients show the difference in trend between treated and non-treated, from a linear spline function with a kink point in birth year 2003. H0: Equal Trends = Test of null hypothesis according to which the difference in trends between treated and non-treated is the same before and after 2003. Probability weights adjusted for country population. Standard errors in parentheses clustered at survey cluster (PSU) level. ${ }^{* * *} p<0.01,{ }^{* *} p<0.05,{ }^{*} p<0.10$. 


\section{Appendix Tables}

Table A.1: Survey waves and years

\begin{tabular}{ll}
\hline \hline Country & Survey years \\
\hline Benin & 2001,2011 \\
Burkina Faso & 2003,2010 \\
Cameroon & 2004,2011 \\
Democratic Republic of Congo & 2007,2013 \\
Ghana & $2003,2008,2014$ \\
Guinea & 2005,2012 \\
Kenya & $2003,2008,2014$ \\
Liberia & 2007,2013 \\
Malawi & $2000,2004,2010$ \\
Mali & $2001,2006,2012$ \\
Nigeria & $2003,2008,2013$ \\
Rwanda & 2005,2010 \\
Senegal & $2005,2010,2012$ \\
Sierra Leone & 2008,2013 \\
Uganda & $2000,2006,2011$ \\
Zambia & 2007,2013 \\
\hline
\end{tabular}


Table A.2: Correlation between bednet ownership or use and infant mortality

\begin{tabular}{lcc|cc}
\hline \hline Dep. var. : & \multicolumn{4}{c}{ Died before age 1 } \\
& PSUs averages (between) & Individual level (within) \\
Sample: & Urban & Rural & Urban & Rural \\
& $(1)$ & $(2)$ & $(3)$ & $(4)$ \\
\hline Bednet ownership & $+0.0173^{*}$ & $+0.0269^{* * *}$ & -0.0050 & -0.0030 \\
& $(0.0098)$ & $(0.0063)$ & $(0.0044)$ & $(0.0034)$ \\
$\mathrm{N}$ & 3,360 & 5,988 & 43,006 & 108,132 \\
$\mathrm{~N}$ clusters & 3,360 & 5,988 & 3,349 & 5,982 \\
\hline Bednet use & $+0.0598^{* * *}$ & $+0.0665^{* * *}$ & $-0.0079^{* *}$ & $-0.0106^{* * *}$ \\
& $(0.0186)$ & $(0.0135)$ & $(0.0039)$ & $(0.029))$ \\
N children & - & - & 41,547 & 105,577 \\
N clusters & 3,360 & 5,988 & 3,344 & 5,982 \\
\hline Children controls & \multicolumn{4}{c}{ Mother age at birth, Child sex } \\
Household controls & \multicolumn{2}{c}{ Wealth, Electricity, Radio, Education } \\
Country FE & Yes & Yes & Yes & Yes \\
Birthyear-country FE & No & No & Yes & Yes \\
Cluster FE & No & No & Yes & Yes \\
\hline
\end{tabular}

Sample: Last DHS wave in each country. Children born 1-4 years before survey.

Notes: Linear models. Bednet ownership is a dummy variable for the household owning at least one mosquito bednet. Bednet use is a dummy variable for at least one child under five year-old in the household having slept under a bednet during the previous night. Probability weights adjusted for country population. In the between regressions, all variables are averaged at cluster level, and standard errors are clustered at the country-level. In the within regressions, standard errors are clustered at the cluster (PSU) level. ${ }^{* * *} \mathrm{p}<0.01,{ }^{* *} \mathrm{p}<0.05,{ }^{*} \mathrm{p}<0.10$. 


\section{Online Appendix}

\section{Appendix Figures - Online Only}

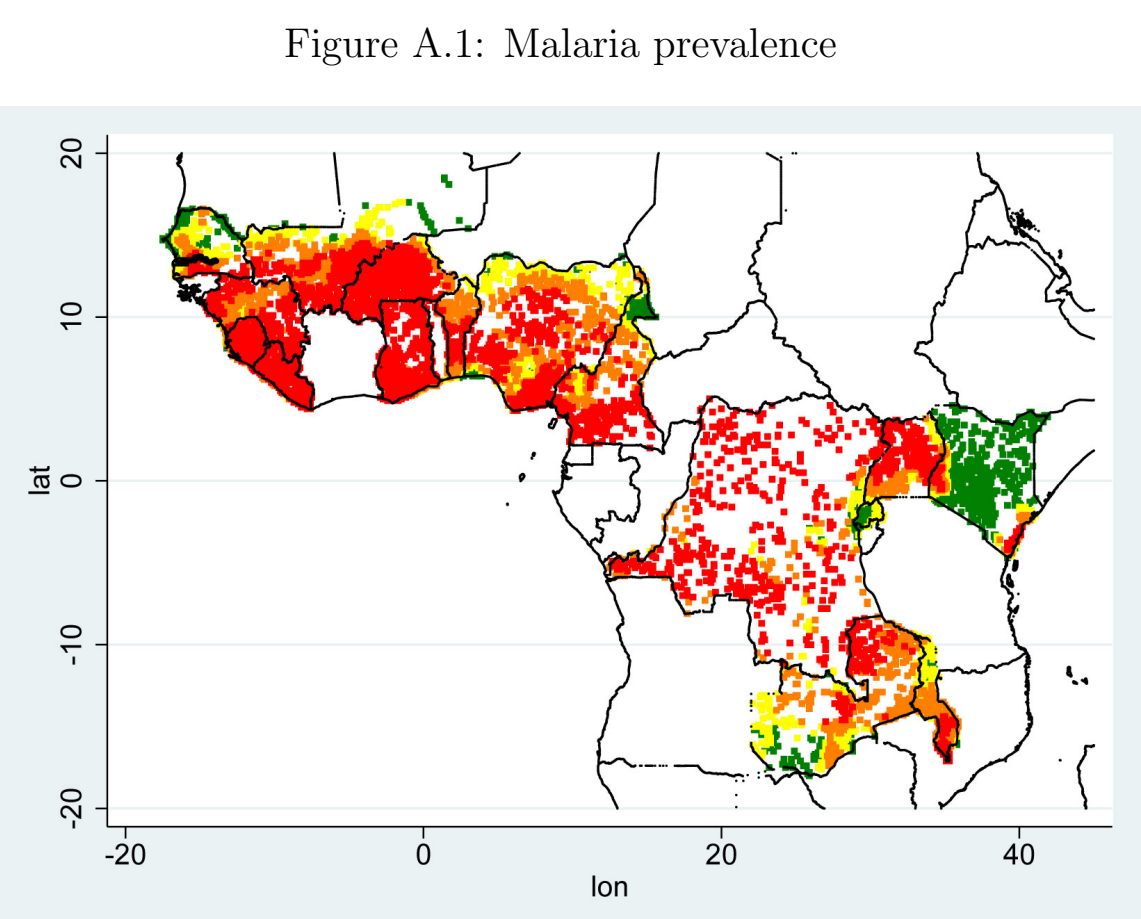

Notes: The share of 2-10 y.o. children infected by Plasmodium falsiparum in the year 2000 is estimated at the 2.5 arcminute (i.e. 0.04 degrees) resolution by the Malaria Atlas map from Oxford University. We matched this map with the spatial coordinates of DHS and computed an average share in a $5 \mathrm{~km}$ (resp. $2 \mathrm{~km}$ ) radius buffer around each rural (resp. urban) cluster, given that DHS coordinates are randomly blurred by $5 \mathrm{~km}$ or $2 \mathrm{~km}$ shifts. The map here shows weighted averages at the 0.1 degrees resolution. Green dots have less than $25 \%$ children infected, yellow dots 25 to $37.5 \%$, orange 37.5 to $50 \%$, and red more than $50 \%$. 
Figure A.2: Double-Difference with Binary Treatment

\section{Rural areas}

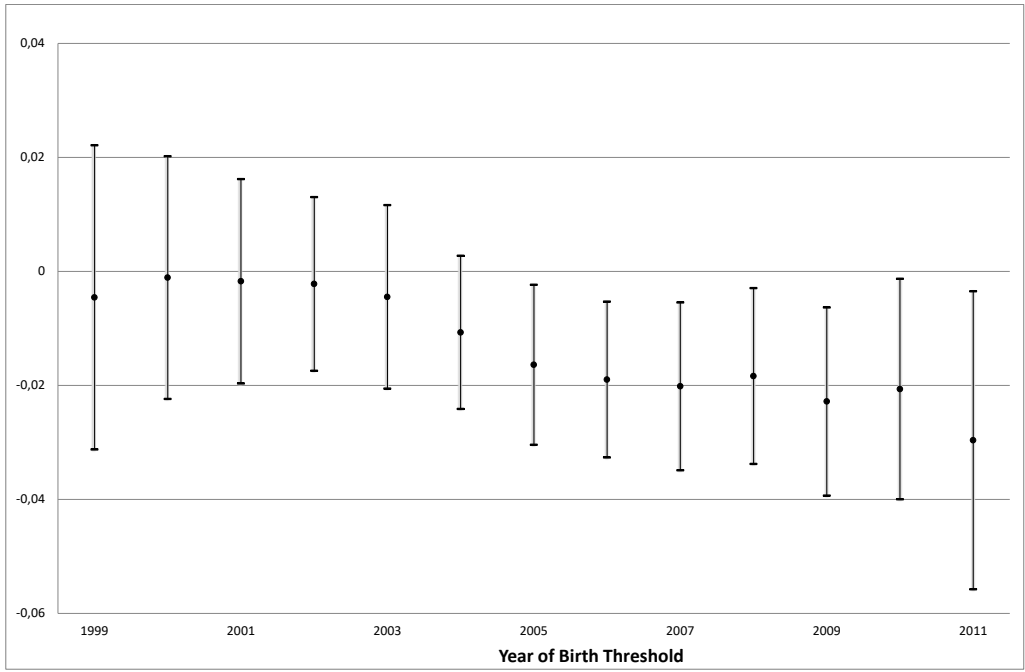

Low Malaria

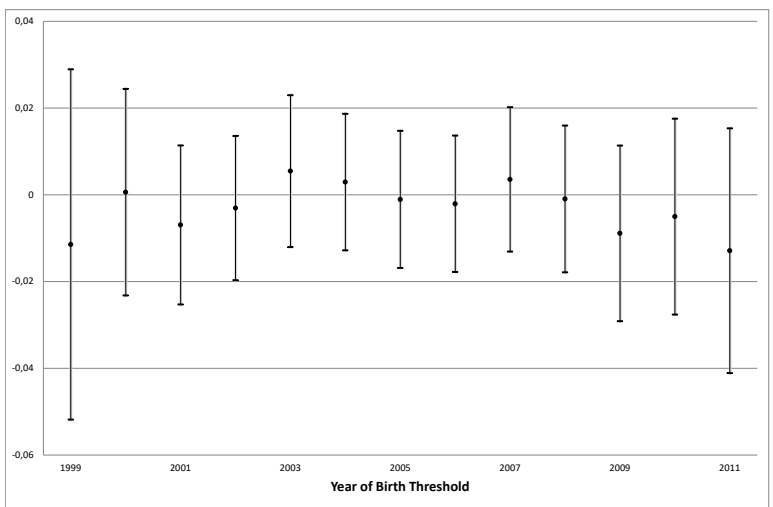

High Malaria

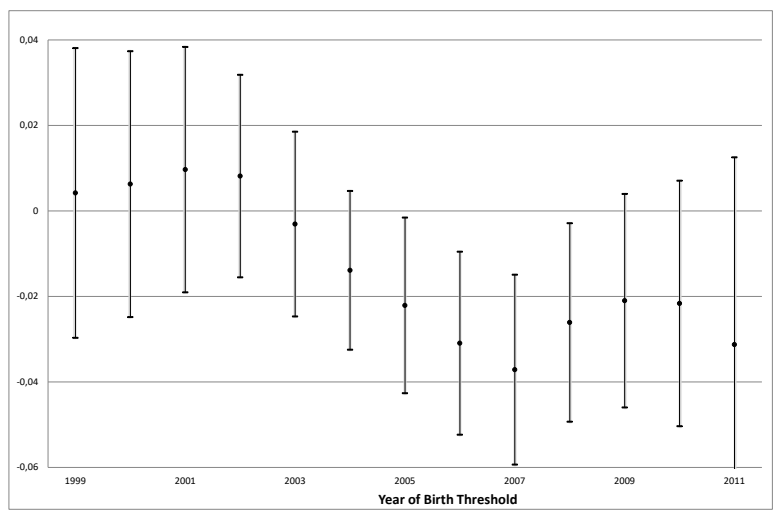

Notes: The graphs display the coefficients of the treatment dummy interacted with a dummy for the year of birth of the child being above a given threshold, with confidence intervals at 95\% (see Equation (4)). Estimation is implemented on the same sample as in Table 7, includes cluster fixed effects, and the same controls. 


\section{Appendix Tables - Online Only}

Table A.3: Sample selection by closeness of neighbor $(<10 \mathrm{~km})$

\begin{tabular}{lcc|cc|cc}
\hline \hline & \multicolumn{2}{c}{ Urban } & \multicolumn{2}{c}{ Rural } & \multicolumn{2}{c}{ Rural High } \\
& Non-sel. & Sel. (diff.) & Non-sel. & Sel. (diff.) & Non-sel. & Sel. (diff.) \\
& $(1 \mathrm{a})$ & $(1 \mathrm{~b})$ & $(2 \mathrm{a})$ & $(2 \mathrm{~b})$ & $(3 \mathrm{a})$ & $(3 \mathrm{~b})$ \\
\hline Distance biggest city $(\mathrm{km})$ & 499.9 & $-96.1^{* * *}$ & 563.1 & $-43.3^{* * *}$ & 524.9 & $-36.5^{* * *}$ \\
Pop. nearest city ('000) & 986.0 & $+118.0^{* * *}$ & 187.9 & +50.9 & 193.6 & +66.2 \\
Malaria prevalence 2000 & 0.471 & $-0.139^{* * *}$ & 0.494 & +0.000 & 0.579 & +0.004 \\
Bednet coverage & 0.660 & $-0.053^{* * *}$ & 0.711 & $+0.022^{*}$ & 0.724 & -0.015 \\
DTP3 Vaccine coverage & 0.615 & $+0.058^{* * *}$ & 0.484 & $+0.059^{* * *}$ & 0.497 & $+0.040^{* *}$ \\
Prenatal care & 0.899 & $+0.046^{* * *}$ & 0.754 & $+0.057^{* * *}$ & 0.793 & $+0.034^{* * *}$ \\
Neonatal mortality & 0.036 & -0.003 & 0.042 & -0.002 & 0.041 & -0.001 \\
Infant mortality & 0.068 & $-0.010^{* * *}$ & 0.084 & -0.002 & 0.083 & -0.001 \\
Wealth index (0-7) & 3.140 & $+0.547^{* * *}$ & 1.784 & $+0.250^{* * *}$ & 1.859 & $+0.220^{* * *}$ \\
Access to electricity & 0.590 & $+0.208^{* * *}$ & 0.156 & $+0.074^{* * *}$ & 0.154 & $+0.073^{* * *}$ \\
Radio ownership & 0.469 & $+0.050^{* *}$ & 0.339 & $+0.054^{* * *}$ & 0.345 & $+0.037^{* * *}$ \\
Mother non-educated & 0.262 & $-0.075^{* * *}$ & 0.527 & $-0.102^{* * *}$ & 0.505 & $-0.082^{* * *}$ \\
Husband education (0-3) & 1.396 & $+0.180^{* * *}$ & 0.847 & $+0.133^{* * *}$ & 0.941 & $+0.114^{* * *}$ \\
\hline Country FE & & Yes & & Yes & & Yes \\
N children & 50,060 & 78,090 & 241,470 & 88,248 & 169,619 & 46,198 \\
N clusters & 1,132 & 2,233 & 4,101 & 1,890 & 2,823 & 859 \\
\hline
\end{tabular}

Sample: All survey clusters in last wave of survey. Children of mothers 15-49 y.o., born not earlier than 5 years before the first survey date.

Notes: Sel. = cluster has two nearest neighbors in first and last wave no further than $10 \mathrm{~km}$. Rural High means rural areas with malaria prevalence above $37.5 \%$ in 2000 according to Malaria Atlas (Bhatt et al., 2015). Pop. nearest city: population of the closest city with more than 10,000 inhabitants at the last available population census. The oldest census date is 1987 for Mali, the latest is 2008 for Liberia, Malawi and Uganda. All variables until prenatal care: weighted averages at cluster level (on mothers 15-49 y.o.). All variables starting with neonatal mortality: weighted average on children. Coefficients reported in columns (1b), (2b) and (3b) are the difference in mean between selected and non-selected, once the country FE has been absorbed. Probability weights adjusted for country population. Standard errors in parentheses clustered at cluster level. *** $p<0.01,{ }^{* *} p<0.05,{ }^{*} p<0.10$. 
Table A.4: Countries in rural, high malaria, selected and treated samples

\begin{tabular}{lcccc}
\hline & Rural & Selected & High Malaria & Treated \\
\hline Benin & 2.73 & 10.72 & 13.37 & 17.77 \\
Burkina Faso & 4.47 & 3.90 & 8.48 & 4.42 \\
Cameroon & 4.29 & 3.62 & 0.77 & 0.00 \\
Democratic Republic of Congo & 14.60 & 0.61 & 0.00 & 0.00 \\
Ghana & 3.96 & 1.54 & 3.35 & 2.61 \\
Guinea & 2.96 & 1.26 & 2.16 & 0.62 \\
Kenya & 8.31 & 20.96 & 18.92 & 27.28 \\
Liberia & 0.56 & 0.90 & 1.96 & 1.41 \\
Malawi & 3.57 & 14.67 & 18.78 & 16.32 \\
Mali & 4.08 & 1.26 & 2.47 & 2.52 \\
Nigeria & 31.44 & 5.46 & 8.91 & 9.63 \\
Rwanda & 3.07 & 19.75 & 0.00 & 0.00 \\
Senegal & 1.92 & 1.69 & 0.00 & 0.00 \\
Sierra Leone & 1.36 & 3.26 & 7.08 & 5.90 \\
Uganda & 9.44 & 9.44 & 12.20 & 11.51 \\
Zambia & 3.23 & 0.96 & 1.56 & 0.00 \\
All & 100.00 & 100.00 & 100.00 & 100.00 \\
\hline
\end{tabular}

Sample: Children of mothers 15-49 y.o., born not earlier than 5 years before the first survey date. Selected $=$ cluster has two nearest neighbors in first and last wave no further than $10 \mathrm{~km}$. High Malaria = selected rural areas with malaria prevalence above $37.5 \%$ in 2000 according to Malaria Atlas (Bhatt et al., 2015). Treated = Among selected highly malarious rural areas, nearest neighbor $(<10 \mathrm{~km})$ in last wave of survey has bednet coverage above $75 \%$, when nearest neighbor in first wave $(<10 \mathrm{~km})$ is below $75 \%$. 
Table A.7: Impact on fertility and neonatal mortality in highly malarious rural areas

\begin{tabular}{lcc|cc}
\hline \hline Dep. var.: & \multicolumn{2}{c}{ Gave birth to a child } & \multicolumn{2}{c}{ Died before month 1 } \\
Indep. var.: & \multicolumn{2}{c}{ Bednet coverage in nearest neighbor } \\
& $(1)$ & $(2)$ & $(3)$ & $(4)$ \\
\hline Continuous & +0.0078 & +0.0105 & $-0.0197^{* *}$ & $-0.0242^{* *}$ \\
& $(0.0075)$ & $(0.0076)$ & $(0.0085)$ & $(0.0096)$ \\
\hline $25-49 \%$ & +0.0079 & $+0.0090^{*}$ & -0.0008 & +0.0000 \\
& $(0.0053)$ & $(0.0050)$ & $(0.0054)$ & $(0.0053)$ \\
$50-74 \%$ & +0.0050 & +0.0058 & -0.0041 & -0.0054 \\
& $(0.0050)$ & $(0.0051)$ & $(0.0068)$ & $(0.0068)$ \\
$75-100 \%$ & +0.0054 & +0.0076 & $-0.0152^{* *}$ & $-0.0166^{* *}$ \\
& $(0.0061)$ & $(0.0062)$ & $(0.0068)$ & $(0.0073)$ \\
\hline Individual controls & \multicolumn{2}{c}{ Mother age } & Mother age, Child sex \\
Household controls & \multicolumn{1}{c}{ Wealth, Electricity, Radio, Education } \\
Neighbor controls & Closest neighbor avg of hh controls \\
Health policies controls & Yes & Yes & Yes & Yes \\
Birthyear-country FE & Yes & Yes & Yes & Yes \\
Cluster FE & Yes & Yes & Yes & Yes \\
Mother FE & No & Yes & No & Yes \\
N & 233,567 & 233,567 & 50,013 & 46,275 \\
N mothers & 18,328 & 18,328 & 17,266 & 13,528 \\
N clusters & 859 & 859 & 859 & 859 \\
\hline
\end{tabular}

Sample: Rural survey clusters of DHS last wave, with malaria prevalence above $37.5 \%$ in 2000 according to Malaria Atlas (Bhatt et al., 2015), and whose two nearest neighbors in first and last wave and of the same urban/rural status are no further than $10 \mathrm{~km}$.

Notes: Fertility: one data point for each mother-year from age 12 to current age of mother. Neonatal mortality: Children of mothers 15-49 y.o., born not earlier than 5 years before the first survey date. Probability weights adjusted for country population. Standard errors in parentheses clustered at survey cluster level. ${ }^{* * *} \mathrm{p}<0.01,{ }^{* *} \mathrm{p}<0.05,{ }^{*} \mathrm{p}<0.10$. 
Table A.8: Differences between non-treated and treated in rural areas according to neighbor's characteristics in first survey wave

\begin{tabular}{lcc|cc}
\hline \hline & \multicolumn{2}{c}{ Rural Low Malaria } & \multicolumn{2}{c}{ Rural High Malaria } \\
& Non-treated & Treated (diff.) & Non-treated & Treated (diff.) \\
\hline Malaria prevalence 2000 & 0.230 & $+0.037^{* * *}$ & 0.572 & -0.014 \\
Bednet coverage & 0.093 & $+0.058^{* * *}$ & 0.168 & +0.016 \\
DTP3 Vaccine coverage & 0.670 & $-0.054^{* *}$ & 0.610 & -0.055 \\
Prenatal care coverage & 0.874 & +0.006 & 0.906 & -0.045 \\
Neonatal mortality & 0.035 & +0.008 & 0.030 & +0.008 \\
Infant mortality & 0.069 & $+0.023^{* *}$ & 0.083 & +0.025 \\
Wealth index (0-7) & 1.373 & -0.022 & 1.614 & -0.002 \\
Access to electricity & 0.0755 & -0.009 & 0.086 & -0.027 \\
Radio ownership & 0.493 & +0.022 & 0.489 & +0.024 \\
Mother non-educated & 0.309 & -0.007 & 0.506 & +0.053 \\
Husband education (0-3) & 1.026 & -0.017 & 0.907 & $-0.158^{* *}$ \\
Distance biggest city (km) & 226.7 & $+52.2^{* * *}$ & 202.8 & $+50.7^{* * *}$ \\
\hline Country FE & & Yes & & Yes \\
N children & 11,979 & 27,415 & 15,510 & 28,248 \\
N clusters & 292 & 677 & 280 & 530 \\
\hline
\end{tabular}

Sample: Rural survey clusters whose nearest neighbor $(<10 \mathrm{~km})$ in first wave of survey had bednet coverage below $75 \%$. Children of mothers 15-49 y.o., born not earlier than 5 years before the first survey date.

Notes: Treated $=$ nearest neighbor $(<10 \mathrm{~km})$ in last wave of survey has bednet coverage above $75 \%$. Values reported are first wave nearest neighbor averages (on mothers 15-49 y.o. in these clusters); neonatal and infant mortality are for children born respectively $0-3$ and 1-3 years before survey date. Coefficients reported in columns (1b) and (2b) is the difference in mean between selected and non-selected, once the country FE has been absorbed. Probability weights adjusted for country population. Standard errors in parentheses clustered at first wave neighbor level. ${ }^{* * *} p<0.01,{ }^{* *} p<0.05,{ }^{*} p<0.10$. 
Table A.9: Extrapolation of the bednet coverage effect by reweighing

\begin{tabular}{|c|c|c|c|}
\hline Rural: & All & Low Malaria & High Malaria \\
\hline \multicolumn{4}{|c|}{ Panel A: Probit Model for nearest neighbors $<10 \mathrm{~km}$} \\
\hline Dist. biggest city $(100 \mathrm{~km})$ & $-0.0214^{* * *}$ & $-0.0437^{* * *}$ & $-0.0348^{* * *}$ \\
\hline Distance biggest city squared & $+0.0015^{* * *}$ & +0.0018 & $+0.0031^{* * *}$ \\
\hline Wealth index $(0-7)$ & +0.0004 & +0.0023 & +0.0013 \\
\hline Access to electricity & $+0.0491^{* * *}$ & +0.0364 & $+0.0597 * * *$ \\
\hline Radio ownership & +0.0037 & +0.0122 & -0.0002 \\
\hline Mother primary education & $+0.0260 * * *$ & $+0.0609 * * *$ & $+0.0183^{* *}$ \\
\hline Mother secondary education & $+0.0546^{* * *}$ & $+0.0974^{* * *}$ & $+0.0580 * * *$ \\
\hline Husband education (0-3) & $+0.0070^{* *}$ & $+0.0211^{*}$ & +0.0042 \\
\hline Country FE & Yes & Yes & Yes \\
\hline $\mathrm{N}$ children & 329,718 & 113,317 & 193,392 \\
\hline $\mathrm{N}$ clusters & 5,991 & 2,297 & 3,342 \\
\hline \multicolumn{4}{|c|}{ Panel B: Infant mortality model reweighed (Table 3) } \\
\hline \multirow[t]{2}{*}{ Continuous } & $-0.0390^{*}$ & +0.0063 & $-0.0914^{* * *}$ \\
\hline & $(0.0211)$ & $(0.0155)$ & $(0.0268)$ \\
\hline \multirow[t]{2}{*}{$25-49 \%$} & +0.0146 & -0.0051 & +0.0055 \\
\hline & $(0.0112)$ & $(0.0114)$ & $(0.0150)$ \\
\hline \multirow[t]{2}{*}{$50-74 \%$} & -0.0111 & +0.0040 & $-0.0336^{*}$ \\
\hline & $(0.0144)$ & $(0.0098)$ & $(0.0171)$ \\
\hline \multirow[t]{2}{*}{$75-100 \%$} & $-0.0389 * *$ & +0.0080 & $-0.0773 * * *$ \\
\hline & $(0.0161)$ & $(0.0125)$ & $(0.0206)$ \\
\hline $\mathrm{N}$ children & 88,247 & 42,047 & 46,196 \\
\hline $\mathrm{N}$ clusters & 1,890 & 1,031 & 859 \\
\hline Children controls & \multicolumn{3}{|c|}{ Mother age at birth, Child sex } \\
\hline Household controls & \multicolumn{3}{|c|}{ Wealth, Electricity, Radio, Education } \\
\hline Neighbor controls & \multicolumn{3}{|c|}{ Average of household controls, Health policies } \\
\hline Birthyear-country FE & Yes & Yes & Yes \\
\hline Cluster FE & Yes & Yes & Yes \\
\hline
\end{tabular}

Sample: Panel A: All rural survey clusters in last wave of survey; Panel B: Rural survey clusters of DHS last wave whose two nearest neighbors of the same urban/rural status in first and last wave are no further than $10 \mathrm{~km}$. Children of mothers $15-49$ y.o., born not earlier than 5 years before the first survey date.

Notes: Panel A reports marginal effects at the mean of a probit model for the selection variable equal to one if the nearest neighbors in first and last wave are no further than $10 \mathrm{~km}$ (see Table A.3). Panel B reports the results of the estimation of Equation (1), where the sample weights used in Table 3 are divided by the predicted probability of Panel A. This probability is abusively treated as deterministic (no bootstrapping), so that standard errors in panel B are likely to be biased downwards. Standard errors in parentheses clustered at survey cluster level. ${ }^{* * *} p<0.01,{ }^{* *} p<0.05,{ }^{*} p<0.10$. 